\title{
ANALYSIS OF THE FINITE ELEMENT VARIATIONAL CRIMES IN THE NUMERICAL APPROXIMATION OF TRANSONIC FLOW
}

\author{
HARALD BERGER AND MILOSLAV FEISTAUER
}

\begin{abstract}
The paper presents a detailed theory of the finite element approximations of two-dimensional transonic potential flow. We consider the boundary value problem for the full potential equation in a general bounded domain $\Omega$ with mixed Dirichlet-Neumann boundary conditions. In the discretization of the problem we proceed as usual in practice: the domain $\Omega$ is approximated by a polygonal domain, conforming piecewise linear triangular elements are used, and the integrals are evaluated by numerical quadratures. Using a new version of entropy compactification of transonic flow and the theory of finite element variational crimes for nonlinear elliptic problems, we prove the convergence of approximate solutions to the exact physical solution of the continuous problem, provided its existence can be shown.
\end{abstract}

\section{INTRODUCTION}

The investigation of transonic flow represents a very interesting part of fluid dynamics, both from physical and mathematical points of view. The interest resides in specific phenomena in high-speed gas flow and in the character of equations describing transonic flow.

Although transonic flow problems play an extremely important role in the design of high-speed airplanes, turbomachines, and compressors, the fundamental general questions concerning the existence and uniqueness of solutions are still open. Some results in this direction were obtained, e.g., by DiPerna [11], Morawetz [29], and Feistauer, Mandel, and Nečas [15, 16, 17, 18, 32]. The publications $[15,16,32]$ emphasize the importance of the second law of thermodynamics represented as an entropy condition; in [11, 17, 18, 29] the viscosity method is studied.

In contrast to the lack of theoretical results there exists a series of methods for the simulation of various types of transonic flow. Here we shall deal with the numerical solution of the transonic flow model based on the full potential equation.

Most numerical methods for the solution of transonic potential flow use finite differences, upwinding in the density and line relaxation, and often apply

Received by the editor October 25, 1990 and, in revised form, March 19, 1992.

1991 Mathematics Subject Classification. Primary 65N30; Secondary 76M10, 76H05.

Key words and phrases. Transonic full potential equation, finite element discretization, discrete entropy compactification, variational crimes.

The first author's research was supported by a grant from the Stiftung Volkswagenwerk, F. R. Germany. 
multigrid techniques $([1,8,25])$. As an extension of this approach, the finite element method on structured meshes combined again with upwinding in the density and line relaxation can be considered $([9,10])$.

Remarkable results were obtained by Glowinski, Pironneau, Bristeau, Periaux, Perrier, and Poirier $([24,5,22,23])$, who use the finite element method on unstructured meshes, least squares, and conjugate gradients. The entropy condition, which is incorporated into the minimization problem as a penalty functional, separates physical solutions from unphysical ones $([24,4])$. The convergence of this method for the case of polygonal domains was proved in Berger [3].

In this paper we shall study the finite element approximation of the transonic flow problem in a general bounded plane domain $\Omega$ described by the full potential equation with mixed Dirichlet-Neumann boundary conditions. In the discretization of the problem we proceed as usual in practice: the domain $\Omega$ with a piecewise curved boundary is approximated by a polygonal one, conforming piecewise linear triangular elements are used, and the integrals are evaluated by numerical quadratures. This means, according to Strang ([34]), that we commit the fundamental variational crimes. In order to improve the results of numerical calculations, we introduce a more involved version of the entropy condition.

Using Berger's generalization ([3]) of the entropy compactification results obtained by Feistauer, Mandel, and Nečas ([15, 16, 27]), and the theory of finite element variational crimes for nonlinear elliptic problems by Feistauer, Ženíšek, Sobotíková $([19,20,21])$, we shall present a detailed analysis for the convergence of entropic approximate solutions to an exact physical solution of the transonic potential flow problem. Special attention will be paid to the complete investigation of the convergence of the least squares method with entropic penalization.

\section{CONTINUOUS PROBLEM}

1.1. Some fundamental concepts. We shall deal with two-dimensional models of stationary, adiabatic, homentropic, compressible, irrotational flows described by the full potential equation

$$
\sum_{i=1}^{2} \frac{\partial}{\partial x_{i}}\left(b(x) \rho\left(|\nabla u|^{2}\right) \frac{\partial u}{\partial x_{i}}\right)=0 \quad \text { in } \Omega .
$$

Here, $\Omega \subset \mathbb{R}^{2}$ is a bounded domain that represents the region filled by the fluid, $u$ is the velocity potential, and $\rho$ is the density given by the relation

$$
\rho(s)=\rho_{0}\left(1-\frac{\kappa-1}{2 a_{0}^{2}} s\right)^{1 /(\kappa-1)}, \quad s \in\left[0, \frac{2 a_{0}^{2}}{\kappa-1}\right],
$$

where $\rho_{0}>0$ and $a_{0}>0$ are the density and speed of sound, respectively, at zero velocity, and $\kappa>1$ is the Poisson adiabatic constant. The velocity field is given by $v=\nabla u=\left(\partial u / \partial x_{1}, \partial u / \partial x_{2}\right)$. If the flow is plane, then $b \equiv 1$. In other two-dimensional models (flow in a fluid layer of variable thickness, axially symmetric flow; cf., e.g., $[13,28,1])$ the function $b$ depends on $x=\left(x_{1}, x_{2}\right) \in \Omega$. 
We usually add to equation (1.1.1) mixed Dirichlet-Neumann boundary conditions
(a) $\left.u\right|_{\Gamma_{D}}=u_{D}$
(b) $\left.b(x) \rho\left(|\nabla u|^{2}\right) \frac{\partial u}{\partial n}\right|_{\Gamma_{N}}=q_{N}$

where $\frac{\partial}{\partial n}$ is the derivative in the direction of the outer unit normal to $\partial \Omega$ and $u_{D}, q_{N}$ are given functions. We assume that

$$
\partial \Omega=\bar{\Gamma}_{D} \cup \bar{\Gamma}_{N}, \quad \Gamma_{D} \cap \Gamma_{N}=\varnothing,
$$

where the sets $\Gamma_{D}$ and $\Gamma_{N}$ are formed by a finite number of open arcs (i.e., arcs without their end points) and $\bar{\Gamma}_{D}, \bar{\Gamma}_{N}$ denote the closures of $\Gamma_{D}$ and $\Gamma_{N}$, respectively.

We assume that the velocity potential $u$ is a single-valued function in $\Omega$. This is true, e.g., if the domain $\Omega$ is simply connected. In multiply connected domains (flow past profiles) the situation becomes more complicated, owing to the fact that $u$ is a multivalued function. We must incorporate the so-called Kutta-Joukowski trailing condition. For simplicity we do not consider this case.

The study of the boundary value problem $(1.1 .1),(1.1 .3, \mathrm{a}-\mathrm{b})$ is fraught with difficulties caused by the nonlinearity of equation (1.1.1) and the fact that it is of mixed type: equation (1.1.1) is

$$
\begin{array}{lll}
\text { elliptic for } & |\nabla u|^{2}<\frac{2 a_{0}^{2}}{\kappa+1} \quad \text { (subsonic flow), } \\
\text { parabolic for } & |\nabla u|^{2}=\frac{2 a_{0}^{2}}{\kappa+1} \quad \text { (sonic flow), } \\
\text { hyperbolic for } & |\nabla u|^{2}>\frac{2 a_{0}^{2}}{\kappa+1} \quad \text { (supersonic flow). }
\end{array}
$$

We say that the flow in $\Omega$ is transonic, if there are two nonempty subsets $\Omega_{1}, \Omega_{2} \subset \Omega$ such that the flow is subsonic in $\Omega_{1}$ and supersonic in $\Omega_{2}$. The boundary between $\Omega_{1}$ and $\Omega_{2}$ is not known in advance and depends on the solution of equation (1.1.1). This boundary is usually formed by sonic lines and shock waves (or briefly shocks), characterized by jumps in the velocity and density. This means that the velocity potential $u$ is not continuously differentiable in $\Omega$.

Across the shock $\Gamma$ we consider the Rankine-Hugoniot transition conditions

$$
\begin{aligned}
& \text { (a) }\left.\frac{\partial u}{\partial t}\right|^{-}=\left.\frac{\partial u}{\partial t}\right|^{+} \quad \text { on } \Gamma, \\
& \text { (b) }\left.\rho\left(|\nabla u|^{2}\right) \frac{\partial u}{\partial n}\right|^{-}=\left.\rho\left(|\nabla u|^{2}\right) \frac{\partial u}{\partial n}\right|^{+} \text {on } \Gamma,
\end{aligned}
$$

where - or + denotes the quantities in front of, or behind, the shock wave (with respect to the flow direction), respectively. By vertical bars and $\frac{\partial}{\partial t}$ and $\frac{\partial}{\partial n}$ we denote here the derivatives with respect to the tangential and normal directions to the shock, respectively.

The fact that transonic flow with shock waves is an irreversible process requires the incorporation of the second law of thermodynamics into our model. We express it as the entropy condition

$$
\left.|\nabla u|\right|^{-}>\left.|\nabla u|\right|^{+} \text {on } \Gamma \text {, }
$$


which means that the velocity must decrease across the shock wave.

We remark that the transition across the shock is connected with an increase of the entropy and a rise of the vorticity. Therefore, the model of irrotational and homentropic flows can only be used provided the quantity $|\nabla u|^{2}$ does not exceed $2 a_{0}^{2} /(\kappa+1)$ too much. Then only so-called weak shocks occur, and the entropy increase as well as the vorticity production across the shock are negligible. This allows us to modify the function $\rho(s)$ given in (1.1.2) close to the point $2 a_{0}^{2} /(\kappa+1)$ and to extend it onto the interval $[0,+\infty)$ in such a way that

(a) $\rho \in C^{1}([0,+\infty))$,

(b) $\rho(s)=\rho_{0}\left(1-\frac{\kappa-1}{2 a_{0}^{2}} s\right)^{1 /(\kappa-1)} \quad$ for $s \in\left[0, s_{1}\right]$ with $s_{1} \in\left(\frac{2 a_{0}^{2}}{\kappa+1}, \frac{2 a_{0}^{2}}{\kappa-1}\right)\left(s_{1}\right.$ is close to $\left.\frac{2 a_{0}^{2}}{\kappa-1}\right)$,

(c) $0<\rho_{\infty} \leq \rho(s) \leq \rho_{0}$, $\left|\rho^{\prime}(s)(1+s)\right| \leq c_{0}$, $\rho^{\prime}(s) \leq 0$ for all $s \in[0,+\infty)$.

Here, $\rho_{0}, \rho_{\infty}, c_{0}>0$ are constants.

Of course, if $u$ is a solution of equation (1.1.1) with the modified density $\rho$ satisfying (1.1.8), then the corresponding velocity field $v=\nabla u$ has physical sense, provided

$$
|\nabla u|^{2} \leq s_{1}
$$

1.2. Formulation of the problem. In the following we shall assume that the boundary $\partial \Omega$ is Lipschitz continuous and piecewise of class $C^{3}$. By the symbols $C^{k}(\bar{\Omega}), C^{\infty}(\bar{\Omega}), L^{p}(\Omega), L^{p}(\partial \Omega), W^{k, p}(\Omega)$, etc. we shall denote the well-known spaces of continuously differentiable functions, Lebesgue and Sobolev spaces of measurable functions, equipped with their usual norms (see, e.g., $[26,31,6])$. We set $C_{0}^{\infty}(\Omega)=\left\{v \in C^{\infty}(\bar{\Omega})\right.$; supp $\left.v \subset \Omega\right\}$, where $\operatorname{supp} v=\overline{\{x ; v(x) \neq 0\}}$ is the support of $v$. Beside the norm in $W^{1,2}(\Omega)$,

$$
\|u\|_{W^{1,2}(\Omega)}=\left(\int_{\Omega}\left(u^{2}+|\nabla u|^{2}\right) d x\right)^{1 / 2},
$$

we shall use the seminorm

$$
|u|_{W^{1,2}(\Omega)}=\left(\int_{\Omega}|\nabla u|^{2} d x\right)^{1 / 2}
$$

The concept of the above spaces will also be employed for other open sets, and we shall use a similar notation.

By meas we shall denote the two-dimensional Lebesgue measure.

1.2.3. Assumptions on data. (A) There exists a domain $\widetilde{\Omega} \subset \mathbb{R}^{2}$ and constants $b_{1}, b_{2}$ such that

$$
\bar{\Omega} \subset \widetilde{\Omega}, \quad b \in C^{1}(\widetilde{\Omega}), \quad 0<b_{1} \leq b \leq b_{2}<+\infty \quad \text { in } \widetilde{\Omega} ;
$$


(B) $q_{N}: \bar{\Gamma}_{N} \rightarrow \mathbb{R}^{1}, q_{N} \in L^{\infty}\left(\Gamma_{N}\right), q_{N}$ is piecewise of the class $C^{2}$ on $\bar{\Gamma}_{N}$; if $\Gamma_{D}=\varnothing$, then

$$
\int_{\partial \Omega} q_{N} d S=0 ;
$$

(C) $u_{D}: \bar{\Gamma}_{D} \rightarrow \mathbb{R}^{1}, u_{D}=\left.u^{*}\right|_{\Gamma_{D}}$, where $u^{*} \in W^{1, \infty}\left(\mathbb{R}^{2}\right)$ (the restriction $\left.u^{*}\right|_{\Gamma_{D}}$ is meant to be in the sense of traces on $\partial \Omega$ ); if $\Gamma_{D}=\varnothing$, we set $u^{*} \equiv 0$.

1.2.4. Classical formulation. For simplicity, let us assume that $\Omega$ is a simply connected domain (e.g., in the form of a channel) and that there exists only one shock wave in $\Omega$ represented by a smooth arc $\Gamma$ such that $\Omega-\Gamma=$ $\Omega^{-} \cup \Omega^{+}$, where $\Omega^{-}, \Omega^{+}$are disjoint nonempty domains. Let us consider the flow direction from $\Omega^{-}$to $\Omega^{+}$. Then we can introduce

1.2.5. Definition. A function $u: \bar{\Omega} \rightarrow \mathbb{R}^{1}$ is a classical solution of the transonic flow problem in $\Omega$, if

1. $u \in C^{0}(\bar{\Omega})$,

2. $u \mid \bar{\Omega}^{ \pm} \in C^{2}\left(\bar{\Omega}^{ \pm}\right)$,

3. $u$ satisfies $(1.1 .1)$ in $\Omega^{-} \cup \Omega^{+}$and $(1.1 .3, \mathrm{a}-\mathrm{b})$ on $\partial \Omega$,

4. $u$ satisfies $(1.1 .6, \mathrm{~b})$.

We say that $u$ is a physical solution, if it satisfies (1.1.7) on $\Gamma$ and (1.1.9) in $\Omega^{-} \cup \Omega^{+}$.

Let us note that $(1.1 .6, a)$ is automatically satisfied if (1) and (2) hold.

1.2.6. Weak formulation. Let us introduce the spaces

(a) $\mathscr{V}=\left\{v \in C^{\infty}(\bar{\Omega}) ; \operatorname{supp} v \subset \Omega \cup \Gamma_{N}\right\} \quad$ if $\Gamma_{D} \neq \varnothing$,

(b) $\mathscr{V}=\left\{v \in C^{\infty}(\bar{\Omega}) ; \int_{\Omega} v d x=0\right\} \quad$ if $\Gamma_{D}=\varnothing$

and set

$$
V=\overline{\mathscr{V}}^{W^{1,2}(\Omega)}=\text { the closure of } \mathscr{V} \text { in } W^{1,2}(\Omega) .
$$

1.2.9. Lemma. We have

(a) $V=\left\{v \in W^{1,2}(\Omega) ;\left.v\right|_{\Gamma_{D}}=0\right\}$ if $\Gamma_{D} \neq \varnothing$.

(b) $V=\left\{v \in W^{1,2}(\Omega) ; \int_{\Omega} v d x=0\right\}$ if $\Gamma_{D}=\varnothing$.

The proof of case (a) can be found in [12]; in case (b) we use regularization (cf. [26], [31]).

1.2.10. Lemma. There exists a constant $\hat{c}>0$ such that

$$
\|u\|_{W^{1,2}(\Omega)} \leq \hat{c}|u|_{W^{1,2}(\Omega)} \quad \forall u \in V .
$$

Hence, $|\cdot|_{W^{1,2}(\Omega)}$ is a norm in $V$ equivalent to the norm $\|\cdot\|_{W^{1,2}(\Omega)}$.

The proof follows from Friedrichs' or Poincaré's inequality in the case 1.2.9,

(a) or (b), respectively (cf. [31]).

Further, let us define the forms

$$
\begin{gathered}
a(u, v)=\int_{\Omega} b \rho\left(|\nabla u|^{2}\right) \nabla u \cdot \nabla v d x, \quad u, v \in W^{1,2}(\Omega), \\
L(v)=\int_{\Gamma_{N}} q_{N} v d S, \quad v \in W^{1,2}(\Omega),
\end{gathered}
$$

which are linear with respect to $v$. 
1.2.13. Lemma. There exist constants $c, \alpha>0$ such that for all $u, v, w \in$ $W^{1,2}(\Omega)$

(a) $|a(u, v)| \leq c\|u\|_{W^{1,2}(\Omega)}\|v\|_{W^{1,2}(\Omega)}$,

(b) $a(v, v) \geq \alpha|v|_{W^{1,2}(\Omega)}^{2}$,

(c) $|a(u, w)-a(v, w)| \leq c|u-v|_{W^{1,2(\Omega)} \mid}|w|_{W^{1,2}(\Omega)}$,

(d) $|L(v)| \leq c\|v\|_{W^{1,2(\Omega)}}$.

Hence, the functions $a(u, \cdot) \quad\left(u \in W^{1,2}(\Omega)\right)$ and $L$ are continuous linear functionals on $W^{1,2}(\Omega)$.

The proof follows from properties (1.1.8) of the function $\rho$ and assumptions 1.2.3 (A), (B). See, e.g., [3].

The weak formulation of the problem can be derived in a standard way. Starting from the classical solution in the sense of Definition 1.2.5, we multiply (1.1.1) by an arbitrary $v \in \mathscr{V}$, integrate over $\Omega$, apply Green's theorem, and use $(1.1 .3, b),(1.1 .6, b),(1.2 .8)$, and Lemma 1.2.9. We obtain the following problem: Find $u: \bar{\Omega} \rightarrow \mathbb{R}^{1}$ such that

$$
\begin{aligned}
& \text { (a) } u \in W^{1,2}(\Omega), \\
& \text { (b) } u-u^{*} \in V, \\
& \text { (c) } a(u, v)=L(v) \quad \forall v \in V .
\end{aligned}
$$

We call $u$ a weak solution of problem (1.1.1), (1.1.3, a-b), (1.1.6, a-b).

It is easy to show that problems $1.2 .5(1)-(4)$ and $(1.2 .14, a-c)$ are formally equivalent. This means that the classical solution $u$ satisfies $(1.2 .14, \mathrm{a}-\mathrm{c})$ and conversely, a weak solution satisfying conditions (1), (2) of 1.2.5 is a classical solution. However, the concept of a weak solution is more general, and it can also be used for transonic flow with several shock waves.

1.2.15. Weak formulation of the entropy condition. In order to get a physical weak solution, it is necessary that this solution fulfills condition (1.1.9) and satisfies, in addition, the entropy condition (1.1.7) in a suitable sense.

Glowinski and Pironneau $([24,5,22,23])$ originally suggested an equivalent form of the entropy condition (1.1.7), which reads

$$
-\int_{\Omega} \nabla u \cdot \nabla v d x \leq K \int_{\Omega} v d x \quad \forall v \in C_{0}^{\infty}(\Omega)^{+} .
$$

Here, $K \geq 0$ is a suitable constant and

$$
C_{0}^{\infty}(\Omega)^{+}=\left\{v \in C_{0}^{\infty}(\Omega) ; v \geq 0 \text { in } \Omega\right\} .
$$

This condition has a very strong compactifying property, as has been pointed out in $[15,16,32]$. Nevertheless, its discrete analogue on unstructured meshes causes sometimes instabilities near the solid wall boundary, where the supersonic pocket occurs. Therefore, it was suggested to apply condition (1.2.16) with test functions from $C^{\infty}(\bar{\Omega})^{+}$instead of $C_{0}^{\infty}(\Omega)^{+}$. However, this modification is no longer consistent with the original (1.1.7) and moreover, in our numerical experiments we observed that its discretization causes convergence problems of the numerical scheme on the inflow and outflow boundary.

In order to overcome these various difficulties, we shall develop a new approach, which has good properties both from the theoretical and numerical point of view. 
We assume that the Neumann boundary $\Gamma_{N}$ is split into two parts $\Gamma_{N}^{0}$ and $\Gamma_{N}^{1}$ satisfying

$$
\begin{array}{cl}
\bar{\Gamma}_{N}=\bar{\Gamma}_{N}^{0} \cup \bar{\Gamma}_{N}^{1}, & \Gamma_{N}^{0} \cap \Gamma_{N}^{1}=\varnothing, \\
\left.q_{N}\right|_{\Gamma_{N}^{0}} \equiv 0, & \left.q_{N}\right|_{\Gamma_{N}^{1}} \neq 0 .
\end{array}
$$

We assume that $\Gamma_{N}^{0}$ and $\Gamma_{N}^{1}$ are unions of a finite number of open arcs.

In order to reformulate condition (1.1.7), we introduce the following sets:

$$
\begin{gathered}
\mathscr{E}^{+}=\left\{v \in C^{\infty}(\bar{\Omega}) ; \operatorname{supp} v \subset \Omega \cup \Gamma_{N}^{0}, v \geq 0 \text { in } \Omega\right\}, \\
E^{+}=\overline{\mathscr{E}}^{W^{1,2}(\Omega)} .
\end{gathered}
$$

Using the techniques from [12], we can show that

$$
E^{+}=\left\{v \in W^{1,2}(\Omega) ;\left.v\right|_{\Gamma_{D} \cup \Gamma_{N}^{1}}=0, v \geq 0 \text { in } \Omega\right\}
$$

Now the modified entropy condition has the form

$$
-\int_{\Omega} \nabla u \cdot \nabla v d x \leq K \int_{\Omega} v d x \quad \forall v \in \mathscr{C}^{+}
$$

In view of (1.2.20), we can also use $v \in E^{+}$as test functions in (1.2.22). Further, it is evident that (1.2.22) implies (1.2.16). If we assume regularity of the solution $u$ and the shock wave $\Gamma$ mentioned in Definition 1.2 .5 , then by virtue of (1.2.18) it is possible to prove the equivalence of (1.2.22) and (1.1.7).

In view of the above considerations we define in $W^{1,2}(\Omega)$ the closed convex subset of admissible functions

$$
\begin{aligned}
\varphi_{s_{1}, K}^{a d}\left\{u \in W^{1, \infty}(\Omega) ;\right. & \|\nabla u\|_{L^{\infty}(\Omega)}^{2} \leq s_{1}, \\
& \left.-\int_{\Omega} \nabla u \cdot \nabla v d x \leq K \int_{\Omega} v d x \forall v \in \mathscr{C}^{+}\right\}
\end{aligned}
$$

and introduce the following

1.2.24. Definition. We say that $u: \bar{\Omega} \rightarrow \mathbb{R}^{1}$ is a weak physical solution of the transonic flow problem, if it satisfies $(1.2 .14, \mathrm{a}-\mathrm{c})$ and, moreover, $u \in \varphi_{s_{1}, K}^{a d}$ for some $K \geq 0$.

\section{DisCRETE PROBLEM}

2.1. Triangulations and finite element spaces. Let us consider systems $\left\{\Omega_{h}\right\}_{h \in\left(0, h_{0}\right)}\left(h_{0}>0\right)$ of polygonal approximations of $\Omega$ and triangulations $\left\{\mathscr{T}_{h}\right\}_{h \in\left(0, h_{0}\right)}$ of $\Omega_{h}$ with the following properties:

(a) the vertices of $\Omega_{h}$ lie on $\partial \Omega$,

(b) $\mathscr{T}_{h}$ is formed by a finite number of closed triangles,

(c) $\bar{\Omega}_{h}=\bigcup_{T \in \mathscr{T}_{h}} T$,

(d) if $T_{1}, T_{2} \in \mathscr{T}_{h}, T_{1} \neq T_{2}$, then either $T_{1} \cap T_{2}=\varnothing$ or $T_{1} \cap T_{2}$ is a common vertex or a common side of $T_{1}, T_{2}$,

(e) if $T \in \mathscr{T}_{h}$, then at most two vertices of $T$ lie on $\partial \boldsymbol{\Omega}$. 
We denote by $\sigma_{h}=\left\{P_{1}, \ldots, P_{N_{h}}\right\}$ the set of all vertices of $\mathscr{T}_{h}$ and let

(a) $\sigma_{h} \subset \bar{\Omega}, \sigma_{h} \cap \partial \Omega_{h} \subset \partial \Omega$,

(b) $\bar{\Gamma}_{D} \cap \bar{\Gamma}_{N}, \bar{\Gamma}_{N}^{0} \cap \bar{\Gamma}_{N}^{1} \subset \sigma_{h}$,

(c) the points of $\partial \Omega$, where either the condition of $C^{3}$ smoothness of $\partial \Omega$ or the condition of $C^{2}$-smoothness of $q_{N}$ are not satisfied, are elements of $\sigma_{h}$.

By $h_{T}$ and $\vartheta_{T}$ we shall denote the length of the maximal side and the magnitude of the minimal angle of $T \in \mathscr{T}_{h}$, respectively. We set

$$
h=\max _{T \in \mathscr{Y}_{h}} h_{T}, \quad \vartheta_{h}=\min _{T \in \mathscr{Y}_{h}} \vartheta_{T} .
$$

We shall assume that the system $\left\{\mathscr{T}_{h}\right\}_{h \in\left(0, h_{0}\right)}$ is regular. This means that there exists $\vartheta_{0}$ such that

$$
\vartheta_{h} \geq \vartheta_{0}>0 \quad \forall h \in\left(0, h_{0}\right) .
$$

By $\bar{\Gamma}_{D_{h}}, \bar{\Gamma}_{N_{h}}, \bar{\Gamma}_{N_{h}}^{0}$, and $\bar{\Gamma}_{N_{h}}^{1}$ we denote the approximation of $\bar{\Gamma}_{D}, \bar{\Gamma}_{N}, \bar{\Gamma}_{N}^{0}$, and $\bar{\Gamma}_{N}^{1}$, respectively.

Approximate solutions to problem $(1.2 .14, \mathrm{a}-\mathrm{c})$ will be sought in the finitedimensional space of conforming piecewise linear elements

$$
X_{h}=\left\{v_{h} ; v_{h} \in C^{0}\left(\bar{\Omega}_{h}\right), v_{h} \text { is affine on each } T \in \mathscr{T}_{h}\right\} .
$$

The space $V$ will be approximated by

$$
\text { (a) } \begin{aligned}
V_{h} & =\left\{v_{h} \in X_{h} ;\left.v_{h}\right|_{\Gamma_{h}}=0\right\} \\
& \equiv\left\{v_{h} \in X_{h} ; v_{h}\left(P_{i}\right)=0 \forall P_{i} \in \sigma_{h} \cap \bar{\Gamma}_{D}\right\} \quad \text { if } \Gamma_{D} \neq \varnothing, \\
\text { (b) } \quad V_{h} & =\left\{v_{h} \in X_{h} ; \int_{\Omega_{h}} v_{h} d x=0\right\} \quad \text { if } \Gamma_{D}=\varnothing .
\end{aligned}
$$

By $w_{h_{i}}, i=1, \ldots, N_{h}$, we denote the basis functions in $X_{h}$ with the property $w_{h_{i}}\left(P_{j}\right)=\delta_{i j}, i, j=1, \ldots, N_{h}$.

We further denote by $r_{h}: \sigma_{h} \rightarrow X_{h}$ the operator of the Lagrange interpolation:

$$
r_{h} v \in X_{h}, \quad\left(r_{h} v\right)\left(P_{i}\right)=v\left(P_{i}\right) \quad \forall P_{i} \in \sigma_{h} \quad \text { for } v: \sigma_{h} \rightarrow \mathbb{R}^{1} .
$$

2.2. Finite element discretization of the problem. Let the form $a(u, v)$ be approximated by

$$
\tilde{a}_{h}(u, v)=\int_{\Omega_{h}} b \rho\left(|\nabla u|^{2}\right) \nabla u \cdot \nabla v d x, \quad u, v \in W^{1,2}\left(\Omega_{h}\right) .
$$

In order to approximate $L(v)$, we shall introduce an approximation $q_{N_{h}}: \bar{\Gamma}_{N_{h}} \rightarrow$ $\mathbb{R}^{1}$ of $q_{N}$. Let $x_{1}^{S}, x_{2}^{S}$ be the local Cartesian coordinates in the neighborhood of a side $S \subset \partial \Omega_{h}$ of a triangle $T \in \mathscr{T}_{h}$ adjacent to $\partial \Omega_{h}$ such that $x_{1}^{S}$ and $x_{2}^{S}$ are measured in the tangential and normal direction to $S$, respectively. Now (provided $h \in\left(0, h_{0}\right)$ and $h_{0}$ is sufficiently small), the $\operatorname{arc} \Sigma \subset \partial \Omega$ approximated by the side $S$, can be expressed by the graph of a function $x_{2}^{S}=$ 
$\varphi_{h}^{S}\left(x_{1}^{S}\right), x_{1}^{S} \in[0, s(S)](s(S)=$ the length of $S)$. Then for $S \subset \bar{\Gamma}_{N_{h}}$ and $\Sigma \subset \bar{\Gamma}_{N}$ we define $q_{N_{h}} \mid S$ as

$$
q_{N_{h}}\left(x_{1}^{S}\right)=q_{N}\left(x_{1}^{S}, \varphi_{h}^{S}\left(x_{1}^{S}\right)\right)
$$

and put

$$
\widetilde{L}_{h}\left(v_{h}\right)=\int_{\Gamma_{N_{h}}} q_{N_{h}} v d S, \quad v \in W^{1,2}\left(\Omega_{h}\right) .
$$

We note that from the assumption that $\partial \Omega$ is Lipschitz continuous and piecewise of class $C^{3}$ it follows that (cf. $[14, \S 3.3]$ )

$$
\left|\varphi_{h}^{S}\left(x_{1}^{S}\right)\right| \leq c h^{2}, \quad x_{1}^{S} \in[0, s(S)] .
$$

Here and in the sequel, $c$ denotes a generic constant independent of $h$, which can have different values at different places.

From the Sobolev imbedding theorems $([6,26,31])$ it follows that

$$
W^{1, p}(\Omega) \subset C^{0}(\bar{\Omega}) \text { for } p \in(2, \infty] \text {. }
$$

Therefore, each $v \in W^{1, r}(\Omega)$ is for $r>2$ defined on the set $\sigma_{h}$, and we can construct its Lagrange interpolant $r_{h} v$.

The approximation of the Dirichlet boundary data $u^{*} \in W^{1, \infty}\left(\mathbb{R}^{2}\right)$ is given by

$$
u_{h}^{*}=r_{h} u^{*} \in X_{h} .
$$

Now we could already formulate the discrete problem of $(1.2 .14$, a-c). However, in practice the integrals in (2.2.1) and (2.2.3) are evaluated by numerical integration. We write

$$
\begin{aligned}
& \text { (a) } \int_{\Omega_{h}} F d x=\sum_{T \in \mathscr{T}_{h}} \int_{T} F d x, \\
& \text { (b) } \int_{T} F d x \approx \operatorname{meas}(T) \sum_{k=1}^{k_{T}} \omega_{T, k} F\left(x_{T, k}\right) \quad \text { if } F \in C^{0}(T) .
\end{aligned}
$$

Here, $x_{T, k} \in T$ and $\omega_{T, k} \in \mathbb{R}^{1}$. We shall assume that

$$
\begin{aligned}
& \text { (a) } \omega_{T, k}>0, \\
& \text { (b) } \sum_{k=1}^{k_{T}} \omega_{T, k}=1 .
\end{aligned}
$$

Similarly, we evaluate integrals over $\Gamma_{N_{h}}$ :

$$
\begin{aligned}
& \text { (a) } \int_{\Gamma_{N_{h}}} F d S=\sum_{S \subset \bar{\Gamma}_{N_{h}}} \int_{S} F d S, \\
& \text { (b) } \int_{S} F d S=s(S) \sum_{j=1}^{k_{S}} \beta_{S, j} F\left(x_{S, j}\right) \quad \text { if } F \in C^{0}(S),
\end{aligned}
$$

where $x_{S, j} \in S, \beta_{S, j} \in \mathbb{R}^{1}$ and $S \subset \bar{\Gamma}_{N_{h}}$ is a side of a triangle $T \in \mathscr{T}_{h}$. We assume that

$$
\text { the order of the integration formulas }(2.2 .7, b) \text { and }
$$

$$
(2.2 .9, b) \text { is } \geq 1 \text {. }
$$


If we approximate the forms $\tilde{a}_{h}$ and $\widetilde{L}_{h}$ by means of $(2.2 .7, \mathrm{a}-\mathrm{b})$ and (2.2.9, a-b), then for $u_{h}, v_{h} \in X_{h}$ we get

$$
\begin{gathered}
\text { (a) } a_{h}\left(u_{h}, v_{h}\right)=\left.\sum_{T \in \mathscr{T}_{h}} \operatorname{meas}(T) \rho\left(\left|\left(\nabla u_{h} \mid T\right)\right|^{2}\right) \nabla u_{h}\right|_{T} \\
\left.\cdot \nabla v_{h}\right|_{T} \sum_{j=1}^{k_{T}} \omega_{T, j} b\left(x_{T, j}\right), \\
\text { (b) } L_{h}\left(v_{h}\right)=\sum_{S \subset \bar{\Gamma}_{N_{h}}} s(S) \sum_{j=1}^{k_{S}} \beta_{s, j} q_{N_{h}}\left(x_{S, j}\right) v_{h}\left(x_{S, j}\right) .
\end{gathered}
$$

Note that if all $x_{T, j} \in \mathscr{T}_{h}$ and $x_{S, j} \in \mathscr{T}_{h} \cap \bar{\Gamma}_{N}$, then for practical computations it is not necessary to extend the function $b$ outside of $\Omega$ and to introduce the approximation $q_{N_{h}}$ of $q_{N}$.

From the results of [20, Theorems 2.2.4 and 2.2.7] it is known that because of (1.1.8), 1.2.3, (2.2.8), and (2.2.10), the following estimates hold:

$$
\begin{array}{r}
\left|a_{h}\left(u_{h}, v_{h}\right)-\tilde{a}_{h}\left(u_{h}, v_{h}\right)\right| \leq c h\left(1+\left\|u_{h}\right\|_{W^{1,2}\left(\Omega_{h}\right)}\right)\left\|v_{h}\right\|_{W^{1,2}\left(\Omega_{h}\right)} \\
\forall u_{h}, v_{h} \in X_{h}, \forall h \in\left(0, h_{0}\right), \\
\left|L_{h}\left(v_{h}\right)-\widetilde{L}_{h}\left(v_{h}\right)\right| \leq c h\left\|v_{h}\right\|_{W^{1,2}\left(\Omega_{h}\right)} \quad \forall v_{h} \in X_{h}, \forall h \in\left(0, h_{0}\right) .
\end{array}
$$

In view of later considerations we shall add to $L_{h}$ a continuous linear perturbation functional $\mathscr{H}_{h}: V_{h} \rightarrow \mathbb{R}^{1}$ which has the property

$$
\lim _{h \rightarrow 0}\left\{\sup _{\substack{v_{h} \in V_{h} \\ v_{h} \neq 0}} \frac{\left|\mathscr{H}_{h}\left(v_{h}\right)\right|}{\left\|v_{h}\right\|_{W^{1,2}\left(\Omega_{h}\right)}}\right\}=0 .
$$

(Its form will be specified later.)

Now we come to

2.2.15. Discrete problem. Find $u_{h}: \bar{\Omega}_{h} \rightarrow \mathbb{R}^{1}$ such that

(a) $u_{h} \in X_{h}$,

(b) $u_{h}-u_{h}^{*} \in V_{h}$,

(c) $a_{h}\left(u_{h}, v_{h}\right)=L_{h}\left(v_{h}\right)+\mathscr{H}_{h}\left(v_{h}\right) \quad \forall v_{h} \in V_{h}$.

Using the same techniques as in $[19,21]$, we can prove the solvability of problem $(2.2 .16, \mathrm{a}-\mathrm{c})$ :

2.2.17. Theorem. For each $h \in\left(0, h_{0}\right)$ there exists at least one solution $u_{h}$ of problem $(2.2 .16, \mathrm{a}-\mathrm{c})$ which satisfies the estimate

$$
\left\|u_{h}\right\|_{W^{1,2}\left(\Omega_{h}\right)} \leq c, \quad h \in\left(0, h_{0}\right),
$$

with $c$ independent of $h$.

In the discretization of the entropy condition (1.2.22) we can proceed in a natural way, leading to

$$
\begin{aligned}
& -\int_{\Omega_{h}} \nabla u_{h} \cdot \nabla v_{h} d x \leq K \int_{\Omega_{h}} v_{h} d x \\
& \quad \forall v_{h} \in E_{h}^{+}=\left\{v_{h} \in X_{h} ;\left.v_{h}\right|_{\bar{\Gamma}_{D_{h}} \cup \overline{\mathrm{N}}_{N_{h}}^{1}}=0 \text { and } v_{h} \geq 0 \text { in } \bar{\Omega}_{h}\right\} .
\end{aligned}
$$


However, it will be convenient to generalize this condition in the following way:

2.2.19. Definition. Let $K \geq 0$ be an arbitrary fixed constant. A function $u_{h} \in X_{h}$ satisfies the discrete entropy condition, if

$$
-\int_{\Omega_{h}} \nabla u_{h} \cdot \nabla v_{h} d x \leq K \int_{\Omega_{h}} v_{h} d x+\gamma(h)\left\|v_{h}\right\|_{L^{\infty}\left(\Omega_{h}\right)} \quad \forall v_{h} \in E_{h}^{+},
$$

where $\gamma:\left(0, h_{0}\right) \rightarrow[0,+\infty)$ and

$$
\lim _{h \rightarrow 0_{+}} \gamma(h)=0 \text {. }
$$

2.2.22. Definition. We say that $u_{h}: \bar{\Omega}_{h} \rightarrow \mathbb{R}^{1}$ is a physical approximate solution of the transonic flow problem if it satisfies $\left(2.2 .16\right.$, a-c) (where $\mathscr{H}_{h}$ has property (2.2.14)) and

$$
\begin{array}{r}
u_{h} \in \varphi_{s_{1}, K, h}^{a d}:=\left\{\varphi_{h} \in X_{h} ;\left\|\nabla \varphi_{h}\right\|_{L^{\infty}\left(\Omega_{h}\right)}^{2} \leq s_{1} \text { and } \varphi_{h}\right. \text { satisfies } \\
\text { the discrete entropy condition(2.2.20) }\} .
\end{array}
$$

Let us remark that Theorem 2.2.17 does not guarantee the existence of a solution that satisfies, in addition, the discrete entropy condition and the uniform pointwise estimate for the gradient. Therefore, we shall reformulate the discrete problem in a suitable way which takes these features into account.

2.3. Least squares method with penalization. In this subsection we shall reformulate the problem of finding a physical approximate solution as a minimization problem. This approach was introduced by Glowinski and Pironneau in their paper [24]. (Cf. also [3, 4, 5, 22, 23].) Instead of seeking the solution $u_{h}$ of $(2.2 .16, \mathrm{a}-\mathrm{c})$ satisfying (2.2.23), we shall solve an appropriate minimization problem. set

Let us denote $A_{i}=\operatorname{meas}\left(\operatorname{supp} w_{h_{i}}\right), i=1, \ldots, N_{h}$. Further, we define the

$$
\hat{\sigma}_{h}=\left\{P_{i} \in \sigma_{h}: P_{i} \notin \bar{\Gamma}_{D} \cup \bar{\Gamma}_{N}^{1}\right\} .
$$

Now we introduce the positive functional $\mathscr{I}_{h}: X_{h} \rightarrow \mathbb{R}^{1}$ by

$$
\begin{aligned}
\mathscr{I}_{h}\left(\varphi_{h}\right):= & \frac{1}{2} \int_{\Omega_{h}}\left|\nabla \xi_{h}\left(\varphi_{h}\right)\right|^{2} d x \\
& +\frac{\mu}{2} \sum_{P_{i} \in \hat{\sigma}_{h}} \frac{1}{A_{i}^{\varepsilon}}\left[\left(-\int_{\Omega_{h}} \nabla \varphi_{h} \cdot \nabla w_{h_{i}} d x-K \int_{\Omega_{h}} w_{h_{i}} d x\right)^{+}\right]^{2},
\end{aligned}
$$

where $\mu>0, K \geq 0, \varepsilon>0$ are arbitrary but fixed constants and $(t)^{+}=$ $\max (t, 0)$ for $t \in \mathbb{R}^{1}$. The function $\xi_{h}:=\xi_{h}\left(\varphi_{h}\right)$ is defined as a solution of the discrete weak Poisson problem

$$
\begin{aligned}
& \text { (a) } \xi_{h} \in V_{h}, \\
& \text { (b) } \int_{\Omega_{h}} \nabla \xi_{h} \cdot \nabla v_{h} d x=a_{h}\left(\varphi_{h}, v_{h}\right)-L_{h}\left(v_{h}\right) \quad \forall v_{h} \in V_{h} .
\end{aligned}
$$


The functional $\mathscr{I}_{h}$ can be considered as a cost functional with (2.3.3) as the corresponding state equation. Note that the function $\xi_{h}\left(\varphi_{h}\right)$ is a weighted residual of the expression $a_{h}\left(\varphi_{h}, v_{h}\right)-L_{h}\left(v_{h}\right)$. Clearly, if $\mathscr{J}_{h}\left(\varphi_{h}\right)=0$ for some $\varphi_{h} \in u_{h}^{*}+V_{h}:=\left\{u_{h} \in X_{h} ; u_{h}=u_{h}^{*}+z_{h}, z_{h} \in V_{h}\right\}$, then $\xi_{h}\left(\varphi_{h}\right)=0$, and $\varphi_{h}$ is a solution of $(2.2 .16, \mathrm{a}-\mathrm{c})$ with $\mathscr{H}_{h} \equiv 0$.

In order to incorporate the discrete entropy condition (2.2.18), Glowinski and Pironneau introduced the penalty functional $\mathscr{P}_{h}: X_{h} \rightarrow \mathbb{R}^{1}$

$$
\mathscr{P}_{h}\left(\varphi_{h}\right):=\frac{\mu}{2} \sum_{P_{i} \in \hat{\sigma}_{h}} \frac{1}{A_{i}^{\varepsilon}}\left[\left(-\int_{\Omega_{h}} \nabla \varphi_{h} \cdot \nabla w_{h_{i}} d x-K \int_{\Omega_{h}} w_{h_{i}} d x\right)^{+}\right]^{2} .
$$

However, they considered the case $\varepsilon \equiv 1$ and $\hat{\sigma}_{h}=\sigma_{h} \cap \Omega_{h}$. It had been observed in Berger's paper [3] that the case $\varepsilon>1$ leads to a convergence problem, provided $\Omega$ is a polygonal domain. Without the incorporation of $\mathscr{P}_{h}$ into the functional $\mathscr{I}_{h}$ one obtains nonphysical solutions with expansion shocks as a minimizer of $\mathscr{I}_{h}$ (see Glowinski and Pironneau [24] and Berger, Warnecke, and Wendland [4]).

We now define a family of functions $\left\{u_{h}\right\}_{h \in\left(0, h_{0}\right)}$ by the solution of the discrete minimization problem

$$
\mathscr{I}_{h}\left(u_{h}\right):=\min _{\substack{\varphi_{h} \in u_{h}^{*}+V_{h} \\\left\|\nabla \varphi_{h}\right\|_{L^{\infty}\left(\Omega_{h}\right)}^{2} \leq s_{2}}} \mathscr{I}_{h}\left(\varphi_{h}\right)
$$

Here, $s_{2} \in[0, \infty)$ is an arbitrary but fixed constant. The functional $\mathscr{J}_{h}$ is continuous, and its minimum over the finite-dimensional closed bounded set $K_{s_{2}, h}:=\left\{\varphi_{h} ; \varphi_{h} \in u_{h}^{*}+V_{h},\left\|\nabla \varphi_{h}\right\|_{L^{\infty}\left(\Omega_{h}\right)}^{2} \leq s_{2}\right\}$ is obtained in at least one element $u_{h} \in K_{s_{2, h}}$.

For our further considerations we shall introduce the following definition and mention some results which will be used later.

2.3.6. Definition. We say that the family $\left\{\mathscr{F}_{h}\right\}$ of triangulations is quasiuniform if each triangle $T \in \mathscr{T}_{h}$ contains a circle of radius $c_{1} h$ and if it is contained in a circle of radius $c_{2} h$. Here, $c_{1}$ and $c_{2}$ are constants independent of $h$ and $T$.

The following estimates can be derived for a quasiuniform family of triangulations (cf., e.g., [6]):

$$
\begin{gathered}
\left\|\nabla w_{h_{i}}\right\|_{L^{\infty}\left(\Omega_{h}\right)} \leq \frac{c}{h}, \quad i=1, \ldots, N_{h}, \\
c h^{2} \leq A_{i} \leq C h^{2}, \quad i=1, \ldots, N_{h}, \\
N_{h} \leq \frac{c}{h^{2}} .
\end{gathered}
$$

Here, $c, C$ are positive constants independent of $h$.

Now we shall prove that the family $\left\{u_{h}\right\}$ defined by (2.3.5) satisfies an estimate which already looks similar to the entropy condition (2.2.20). The technique of the proof is similar to the one for Theorem 4.1 in Berger [3].

2.3.10. Theorem. Let $\left\{\mathscr{T}_{h}\right\}_{h \in\left(0, h_{0}\right)}$ be a quasiuniform family of triangulations, or more generally, let $\left\{\mathscr{T}_{h}\right\}$ be such that (2.3.9) holds. Then the family 
$\left\{u_{h}\right\}_{h \in\left(0, h_{0}\right)}$ of solutions of the minimization problem (2.3.5) satisfies the inequality

$$
\begin{aligned}
-\int_{\Omega_{h}} \nabla u_{h} \cdot \nabla v_{h} d x \leq K \int_{\Omega_{h}} v_{h} d x+c \sqrt{\mathscr{P}_{h}\left(u_{h}\right)} h^{\varepsilon-1}\left\|v_{h}\right\|_{L^{\infty}\left(\Omega_{h}\right)} & \\
& \forall v_{h} \in E_{h}^{+} .
\end{aligned}
$$

(The constant $c>0$ is independent of $h$.)

Proof. Let $v_{h} \in E_{h}^{+}$. Then, by the definition of $\mathscr{P}_{h}\left(u_{h}\right)$ and the Cauchy inequality, we obtain

$$
\begin{aligned}
& -\int_{\Omega_{h}} \nabla u_{h} \cdot \nabla v_{h} d x-K \int_{\Omega_{h}} v_{h} d x \\
& \quad=\sum_{P_{i} \in \hat{\sigma}_{h}} v_{h}\left(P_{i}\right)\left(-\int_{\Omega_{h}} \nabla u_{h} \cdot \nabla w_{h_{i}} d x-K \int_{\Omega_{h}} w_{h_{i}} d x\right) \\
& \quad \leq \sum_{P_{i} \in \hat{\sigma}_{h}} v_{h}\left(P_{i}\right)\left(-\int_{\Omega_{h}} \nabla u_{h} \cdot \nabla w_{h_{i}} d x-K \int_{\Omega_{h}} w_{h_{i}} d x\right)^{+} \\
& \quad \leq\left\|v_{h}\right\|_{L^{\infty}\left(\Omega_{h}\right)} \sum_{P_{i} \in \hat{\sigma}_{h}}\left(-\int_{\Omega_{h}} \nabla u_{h} \cdot \nabla w_{h_{i}} d x-K \int_{\Omega_{h}} w_{h_{i}} d x\right)^{+} \\
& \quad \leq\left\|v_{h}\right\|_{L^{\infty}\left(\Omega_{h}\right)} \sqrt{N_{h}}\left\{\sum_{P_{i} \in \hat{\sigma}_{h}} \frac{A_{i}^{\varepsilon}}{A_{i}^{\varepsilon}}\left[\left(-\int_{\Omega_{h}} \nabla u_{h} \cdot \nabla w_{h_{i}} d x-K \int_{\Omega_{h}} w_{h_{i}} d x\right)^{+}\right]^{2}\right\}^{1 / 2} \\
& \quad \leq\left\|v_{h}\right\|_{L^{\infty}\left(\Omega_{h}\right)} \sqrt{N_{h}} \max _{i=1, \ldots, N_{h}} A_{i}^{\varepsilon / 2} \sqrt{\frac{2}{\mu} \mathscr{P}_{h}\left(u_{h}\right) .}
\end{aligned}
$$

Using (2.3.8) and (2.3.9), we immediately obtain (2.3.11).

2.3.12. Remark. If $\Gamma_{D}=\varnothing$ or $u^{*}=0$, then it is easy to prove that $\mathscr{P}_{h}\left(u_{h}\right)$ is uniformly bounded by a constant independent of $h$ (see [3, Lemma 4.1]). Hence, (2.3.11) implies that for these cases

$$
-\int_{\Omega_{h}} \nabla u_{h} \cdot \nabla v_{h} d x \leq K \int_{\Omega_{h}} v_{h} d x+c h^{\varepsilon-1}\left\|v_{h}\right\|_{L^{\infty}\left(\Omega_{h}\right)} \quad \forall v_{h} \in E_{h}^{+}
$$

is valid, and for $\varepsilon>1$ the solutions of the minimization problem (2.3.5) satisfy the discrete entropy condition (2.2.20), (2.2.21).

\section{CONVERGENCE}

Here we shall deal with the convergence of the approximate finite element solutions $u_{h}$ of the transonic flow problem to an exact solution, provided $h \rightarrow$ 0 . The main tools are the compactification properties of the entropy condition (discovered by Feistauer, Mandel, Nečas [15, 16, 27] and generalized by Berger [3]) and the analysis of the finite element variational crimes for nonlinear elliptic problems by Feistauer, Ženíšek, Sobotíková [19, 20, 21].

3.1. Preliminaries. In the sequel we shall consider a family $\left\{\mathscr{T}_{h}\right\}_{h \in\left(0, h_{0}\right)}$ of triangulations of the domains $\Omega_{h}$ and assume that $h_{0}>0$ is sufficiently small. 
We set

$$
\omega_{h}=\Omega-\bar{\Omega}_{h}, \quad \tau_{h}=\Omega_{h}-\bar{\Omega} .
$$

By [14, Lemma 3.3.4],

$$
\operatorname{meas}\left(\omega_{h} \cup \tau_{h}\right) \leq c h^{2} .
$$

If $T \in \mathscr{T}_{h}$ is a boundary element, i.e., an element adjacent to $\partial \Omega_{h}$ with vertices $P_{1}^{T}, P_{2}^{T} \in \partial \Omega$ and $P_{3}^{T} \in \Omega_{h} \cap \Omega$, then by $T^{\text {id }}$ we shall denote the curved triangle with two straight sides $P_{1}^{T} P_{3}^{T}, P_{2}^{T} P_{3}^{T}$ and one curved side $\sum_{T} \subset \partial \Omega$ with endpoints $P_{1}^{T}, P_{2}^{T}$. We call $T^{\text {id }}$ ideal element associated with $T$.

If we replace all boundary triangles in $\mathscr{T}_{h}$ by their associated ideal elements, we obtain the ideal triangulation $\mathscr{T}_{h}^{\text {id }}$ of the domain $\Omega$, associated with $\mathscr{T}_{h}$.

Let $v_{h} \in X_{h}$. We define its natural extension $\bar{v}_{h} \in C\left(\bar{\Omega}_{h} \cup \bar{\Omega}\right)$ as $\bar{v}_{h}=v_{h}$ on $\bar{\Omega}_{h}$ and $\left.\bar{v}_{h}\right|_{T^{\text {id }}}=\left.p\right|_{T^{\text {id }}}$, where $p$ is the polynomial of order $\leq 1$ satisfying $\left.p\right|_{T}=\left.v_{h}\right|_{T}$. It is evident that $\bar{v}_{h} \in W^{1, p}(\Omega)$ for $p \in[1, \infty]$ (cf. Ciarlet [6, Theorem 2.1.1]).

In [20, Lemma 3.3.13], the following estimate for the approximation $\tilde{L}_{h}$ of the form $L$ was proved:

$$
\left|\tilde{L}_{h}\left(v_{h}\right)-L\left(\bar{v}_{h}\right)\right| \leq \operatorname{ch}^{\frac{3}{2}}\left\|v_{h}\right\|_{W^{1,2}\left(\Omega_{h}\right)}, \quad v_{h} \in X_{h}, h \in\left(0, h_{0}\right) .
$$

Further, by a technique analogous to [20, Lemma 3.3.12], we prove that

$$
\begin{gathered}
\left|\bar{v}_{h}\right|_{W^{1, p}\left(\omega_{h} \cup \tau_{h}\right)} \leq c h^{1 / p}\left\|v_{h}\right\|_{W^{1, p}\left(\Omega_{h}\right)}, \\
\left\|\bar{v}_{h}\right\|_{L^{p}\left(\omega_{h} \cup \tau_{h}\right)} \leq c h^{2 / p}\left\|v_{h}\right\|_{W^{1, p}\left(\Omega_{h}\right)} \\
\forall v_{h} \in X_{h}, \forall h \in\left(0, h_{0}\right), \forall p \in[1, \infty] .
\end{gathered}
$$

Here and in the following, the constant $c$ is independent of $h$ and $v_{h}$, but it can depend on $p$ in general. For the proof of (3.1.5) we need the uniform theorem on traces in $W^{1, p}\left(\Omega_{h}\right)$, which is a consequence of similar arguments as in [14, Theorem 3.3.6] and [26, Chapter 6]: For $p \in[1, \infty]$ there exists a constant $c>0$ such that

$$
\|v\|_{L^{p}\left(\partial \Omega_{h}\right)} \leq c\|v\|_{W^{1, p}\left(\Omega_{h}\right)}, \quad v \in W^{1, p}\left(\Omega_{h}\right), h \in\left(0, h_{0}\right) .
$$

\subsubsection{Lemma. We have}

$$
\left\|v_{h}\right\|_{W^{1,2}\left(\Omega_{h}\right)} \leq c\left|v_{h}\right|_{W^{1,2}\left(\Omega_{h}\right)} \quad \forall v_{h} \in V_{h}, \forall h \in\left(0, h_{0}\right)
$$

with $c$ independent of $v_{h}$ and $h$.

Proof. In the case $\Gamma_{D} \neq \varnothing$ (cf. $\left.(2.1 .6, a)\right)$ the assertion is a consequence of the discrete form of Friedrichs' inequality ([35]).

Let $\Gamma_{D}=\varnothing$. Then $V_{h}$ is defined by $(2.1 .6, \mathrm{~b})$. For $v_{h} \in V_{h}$, let us set

$$
\tilde{v}_{h}=\bar{v}_{h}-\frac{1}{\operatorname{meas}(\Omega)} \int_{\Omega} \bar{v}_{h} d x \text {. }
$$

It is evident that $\tilde{v}_{h} \in V($ see $(1.2 .9, \mathrm{~b}))$ and, by virtue of Lemma 1.2.10,

$$
\left\|\tilde{v}_{h}\right\|_{W^{1,2}(\Omega)}^{2} \leq \hat{c}^{2}\left|\tilde{v}_{h}\right|_{W^{1,2}(\Omega)}^{2}
$$

Now, using the relation

$$
\left|\bar{v}_{h}\right|_{W^{1,2}(\Omega)}^{2}=\left|v_{h}\right|_{W^{1,2}\left(\Omega_{h}\right)}^{2}+|\bar{v}|_{W^{1,2}\left(\omega_{h}\right)}^{2}-\left|v_{h}\right|_{W^{1,2}\left(\tau_{h}\right)}^{2},
$$


similar relations for $\left\|\bar{v}_{h}\right\|_{L^{2}(\Omega)}^{2}$ and $\int_{\Omega} \bar{v}_{h} d x$, estimates (3.1.4), (3.1.5), and $(2.1 .6, b)$, we find that

$$
\begin{aligned}
\left\|\tilde{v}_{h}\right\|_{L^{2}(\Omega)}^{2} & \geq \frac{1}{2}\left(\left\|\bar{v}_{h}\right\|_{L^{2}(\Omega)}^{2}-c\left(\int_{\omega_{h} \cup \tau_{h}}\left|\bar{v}_{h}\right| d x\right)^{2}\right) \\
& \geq \frac{1}{2}\left(\left\|v_{h}\right\|_{L^{2}\left(\Omega_{h}\right)}^{2}-c h^{2}\left\|v_{h}\right\|_{W^{1,2}\left(\Omega_{h}\right)}^{2}\right), \\
\left|\tilde{v}_{h}\right|_{W^{1,2}(\Omega)}^{2} & \leq\left|v_{h}\right|_{W^{1,2}\left(\Omega_{h}\right)}^{2}+c h\left\|v_{h}\right\|_{W^{1,2}\left(\Omega_{h}\right)}^{2}, \\
\left|\tilde{v}_{h}\right|_{W^{1,2}(\Omega)}^{2} & \geq\left|v_{h}\right|_{W^{1,2}\left(\Omega_{h}\right)}^{2}-c h\left\|v_{h}\right\|_{W^{1,2}\left(\Omega_{h}\right)}^{2} .
\end{aligned}
$$

From (3.1.10) and (3.1.11) we get

$$
\left(\frac{1}{2}-c h\right)\left\|v_{h}\right\|_{W^{1,2}\left(\Omega_{h}\right)}^{2} \leq \hat{c}^{2}\left(\left|v_{h}\right|_{W^{1,2}\left(\Omega_{h}\right)}^{2}+c h\left\|v_{h}\right\|_{W^{1,2}\left(\Omega_{h}\right)}^{2}\right),
$$

which immediately yields (3.1.8), provided $h \in\left(0, h_{0}\right)$ and $h_{0}>0$ is sufficiently small.

In the same way as in [21] we define the ideal interpolation $I_{h}^{\text {id }}: C^{0}(\bar{\Omega}) \rightarrow$ $W^{1, p}(\Omega)$. The construction of the interpolant $I_{h}^{\text {id }} v$ to $v \in C(\bar{\Omega})$ is described in [21, Definition 5.1.1], and is based on [20, Lemma 3.3.4] and the results from Zlámal [36]. For our further considerations the following property is important.

If $T \in \mathscr{T}_{h}$ is a boundary triangle with vertices $P_{1}^{T}, P_{2}^{T} \in \partial \Omega, T^{\text {id }}$ is the ideal element associated with $T$, and $\Sigma \subset \partial \Omega$ is the curved side of $T^{\text {id }}$ with end points $P_{1}^{T}, P_{2}^{T}$, then we have the implication

$$
v\left(P_{1}^{T}\right)=v\left(P_{2}^{T}\right)=0 \Rightarrow I_{h}^{\text {id }} v=0 \text { on } \Sigma .
$$

If we proceed in the same way as in the proof of [36, Theorem 2], where we set $k=2$ and work in $W^{k, p}(\Omega) \quad(p \in[1, \infty])$ instead of $W^{k, 2}(\Omega)$, we get the estimate

$$
\left\|I_{h}^{\mathrm{id}} \bar{v}_{h}-\bar{v}_{h}\right\|_{W^{1, p}\left(\Omega_{h}\right)} \leq c h\left\|v_{h}\right\|_{W^{1, p}\left(\Omega_{h}\right)} \quad \forall v_{h} \in X_{h} \forall h \in\left(0, h_{0}\right) .
$$

From (3.1.12), (1.2.21), (2.2.18), and the construction of $I_{h}^{\text {id }}$ (see [21, Definition 5.1.1] and [20, Lemma 3.3.4]) we can see that

$$
v_{h} \in E_{h}^{+} \Rightarrow I_{h}^{\text {id }} v_{h} \in E^{+} .
$$

Further, for $v_{h} \in V_{h}$, define $\hat{v}_{h} \in V$ in the following way:

(a) $\hat{v}_{h}=I_{h}^{\text {id }} \bar{v}_{h} \quad$ if $\Gamma_{D} \neq \varnothing$,

(b) $\hat{v}_{h}=\bar{v}_{h}-\frac{1}{\operatorname{meas}(\Omega)} \int_{\Omega} \bar{v}_{h} d x$ if $\Gamma_{D}=\varnothing$. 
Then we have

3.1.16. Lemma. For $p \in[1, \infty]$ there holds

$$
\left\|\hat{v}_{h}-\bar{v}_{h}\right\|_{W^{1, p}(\Omega)} \leq c h\left\|v_{h}\right\|_{W^{1, p}\left(\Omega_{h}\right)} \quad \forall v_{h} \in V_{h}, \forall h \in\left(0, h_{0}\right) .
$$

Proof. Provided $\Gamma_{D} \neq \varnothing$, the assertion (3.1.17) is a consequence of (3.1.13).

Let $\Gamma_{D}=\varnothing$. Then

$$
\begin{aligned}
\left\|\hat{v}_{h}-\bar{v}_{h}\right\|_{W^{1, p}(\Omega)} & =(\operatorname{meas}(\Omega))^{1 / p-1}\left|\int_{\Omega} \bar{v}_{h} d x\right| \\
& =(\operatorname{meas}(\Omega))^{1 / p-1}\left|\int_{\Omega_{h}} v_{h} d x+\int_{\omega_{h}} \bar{v}_{h} d x-\int_{\tau_{h}} v_{h} d x\right|
\end{aligned}
$$

If we use the assumption that $v_{h} \in V_{h}$, which means that

$$
\int_{\Omega_{h}} v_{h} d x=0,
$$

then from the Hölder inequality, (3.1.2) and (3.1.5) we get the estimate

$$
\left\|\hat{v}_{h}-\bar{v}_{h}\right\|_{W^{1, p}(\Omega)} \leq c h^{2}\left\|v_{h}\right\|_{W^{1, p}\left(\Omega_{h}\right)}
$$

with $c$ independent of $v_{h}$ and $h$. Hence, also (3.1.17) holds.

In the sequel, for $v \in W^{1, p}(\Omega)$ we shall denote by $v_{c}$ the extension of $v$. That is, $v_{c} \in W^{1, p}\left(\mathbb{R}^{2}\right),\left.v_{c}\right|_{\Omega}=v$, and

$$
\left\|v_{c}\right\|_{W^{1, p}\left(\mathbf{R}^{2}\right)} \leq \tilde{c}\|v\|_{W^{1, p}(\Omega)},
$$

where $\tilde{c}$ is independent of $v$ and $p$ (cf. [31, Chapter 2, §3.7]).

By [7, Theorem 6], [19, proof of Lemma 3.1.3], and (3.1.4), (3.1.5) we have the following approximation results:

3.1.19. Lemma. Provided $v \in W^{1, p}(\Omega)$, we have
(a) $\left\|v_{c}-r_{h} v\right\|_{W^{1, p}\left(\Omega_{h}\right)} \stackrel{h \rightarrow 0}{\rightarrow} 0$ for $p \in(2, \infty)$,
(b) $\left\|v-\overline{r_{h} v}\right\|_{W^{1, p}(\Omega)} \stackrel{h \rightarrow 0}{\rightarrow} 0$ for $p \in(2, \infty)$,
(c) $\left\|r_{h} v\right\|_{W^{1, p}\left(\Omega_{h}\right)} \leq c\|v\|_{W^{1, p}(\Omega)}$ for $p \in(2, \infty]$,
(d) $\left\|r_{h} v\right\|_{L^{\infty}\left(\Omega_{h}\right)} \leq\|v\|_{L^{\infty}(\Omega)}$,
(e) $\left\|\overline{r_{h} v}\right\|_{W^{1, p}(\Omega)} \leq c\|v\|_{W^{1, p}(\Omega)}$ for $p \in(2, \infty]$,

where $c$ is a constant independent of $v$ and $h \in\left(0, h_{0}\right)$.

Because of later considerations, in addition to the Lagrange interpolation we introduce its modification $\Pi_{h}: \sigma_{h} \rightarrow V_{h}$ defined in the following way: For $v: \sigma_{h} \rightarrow \mathbb{R}^{1}$ we set
(a) $\Pi_{h} v=r_{h} v$ if $\Gamma_{D} \neq \varnothing$,
(b) $\Pi_{h} v=r_{h} v-\frac{1}{\operatorname{meas}\left(\Omega_{h}\right)} \int_{\Omega_{h}} r_{h} v d x$ if $\Gamma_{D}=\varnothing$. 
3.1.22. Lemma. If $p \in(2, \infty)$ and $v \in V \cap W^{1, p}(\Omega)$, then
(a) $\left\|v_{c}-\Pi_{h} v\right\|_{W^{1, p}\left(\Omega_{h}\right)} \stackrel{h \rightarrow 0}{\rightarrow} 0$,
(b) $\left\|v-\overline{\Pi_{h} v}\right\|_{W^{1, p}(\Omega)} \stackrel{h \rightarrow 0}{\rightarrow} 0$,
(c) $\left\|v-\left(\widehat{\Pi_{h}} v\right)\right\|_{W^{1, p}(\Omega)} \stackrel{h \rightarrow 0}{\rightarrow} 0$.

The proof obtains from (3.1.20, a), (3.1.4), (3.1.5), and a similar technique as in Lemma 3.1.16.

Now let us draw our attention to the solutions $u_{h}$ of problem $(2.2 .16, \mathrm{a}-\mathrm{c})$. We assume that $u_{h}^{*}=r_{h} u^{*}$ and $u^{*} \in W^{1, \infty}\left(\mathbb{R}^{2}\right)$. In view of $(2.2 .16, \mathrm{~b})$, the solution $u_{h}$ can be written in the form

$$
u_{h}=u_{h}^{*}+z_{h}, \quad \text { where } z_{h} \in V_{h} .
$$

Similarly as in [19], we define the function $u_{h}^{\prime} \in W^{1,2}(\Omega)$ associated with $u_{h}$ :

$$
u_{h}^{\prime}=\bar{u}_{h}^{*}+\hat{z}_{h},
$$

where $\hat{z}_{h}$ is defined by (3.1.15).

In view of $(3.1 .20, \mathrm{c}$ and $\mathrm{e})$ we have

$$
\left\|u_{h}^{*}\right\|_{W^{1, p}\left(\Omega_{h}\right)},\left\|\bar{u}_{h}^{*}\right\|_{W^{1, p}(\Omega)} \leq c, \quad h \in\left(0, h_{0}\right), p \in[1, \infty] .
$$

Let $s_{2}>0$ and

$$
\left\|\nabla u_{h}\right\|_{L^{\infty}\left(\Omega_{h}\right)} \leq \sqrt{s_{2}}, \quad h \in\left(0, h_{0}\right) .
$$

Then, of course, also

$$
\left\|\nabla \bar{u}_{h}\right\|_{L^{\infty}(\Omega)} \leq \sqrt{s_{2}}, \quad h \in\left(0, h_{0}\right)
$$

and

$$
\left\|u_{h}\right\|_{W^{1, \infty}\left(\Omega_{h}\right)},\left\|\bar{u}_{h}\right\|_{W^{1, \infty}(\Omega)} \leq c, \quad h \in\left(0, h_{0}\right) .
$$

Further, $u_{h}^{\prime}-\bar{u}_{h}=\hat{z}_{h}-\bar{z}_{h}, z_{h}=u_{h}-u^{*}$, and $u_{h}^{\prime}=\bar{u}_{h}+\left(u_{h}^{\prime}-\bar{u}_{h}\right)$. From this, (3.1.17), (3.1.27), (3.1.29) we get the estimates

$$
\begin{aligned}
\left\|z_{h}\right\|_{W^{1, p}\left(\Omega_{h}\right)} & \leq c, \\
\left\|u_{h}^{\prime}-\bar{u}_{h}\right\|_{W^{1, p}(\Omega)} \leq c h\left\|z_{h}\right\|_{W^{1, p}\left(\Omega_{h}\right)} & \leq c h, \quad h \in\left(0, h_{0}\right), p \in[1, \infty],
\end{aligned}
$$

and

$$
\begin{aligned}
\left\|\nabla u_{h}^{\prime}\right\|_{L^{\infty}(\Omega)} & \leq \sqrt{s_{2}}+\left\|u_{h}^{\prime}-\bar{u}_{h}\right\|_{W^{1}, \infty}(\Omega) \\
& \leq \sqrt{s_{2}}+c h, \quad h \in\left(0, h_{0}\right) .
\end{aligned}
$$

Hence,

$$
\left\|u_{h}^{\prime}\right\|_{W^{1, p}(\Omega)} \leq c, \quad h \in\left(0, h_{0}\right), p \in[1, \infty] .
$$

This implies the existence of a subsequence $\left\{u_{h_{n}}\right\}$ and a function $u$ such that

$$
\begin{array}{ll}
h_{n} \rightarrow 0_{+}, & \\
u_{h_{n}}^{\prime}-u & * \text {-weakly in } W^{1, \infty}(\Omega), \\
u_{h_{n}}^{\prime}-u & \text { weakly in } W^{1, p}(\Omega), p \in[1, \infty)
\end{array}
$$


The aim of our following considerations will be to prove strong convergence of $u_{h_{n}}^{\prime}$ and to show that the limit $u$ is a solution of the continuous transonic flow problem.

3.2. Compactification by the discrete entropy condition. The proof of convergence of the approximate solutions $u_{h}$ to a solution of the continuous problem will be based on the following fundamental theorem:

3.2.1. Theorem. Let $q \in(2, \infty)$ and $G_{n} \in\left(W^{1, q}(\Omega)\right)^{\prime} \quad$ (= dual to the space $\left.W^{1, q}(\Omega)\right)$ be a sequence satisfying

$$
G_{n}-G \text { weakly in }\left(W^{1, q}(\Omega)\right)^{\prime}
$$

and

$$
G_{n}(v) \leq L\|v\|_{L^{\infty}(\Omega)} \quad \forall v \in C_{0}^{\infty}(\Omega)^{+},
$$

where $L$ is a constant independent of $v$ and $n$. Then

$$
G_{n} \rightarrow G \text { strongly in }\left(W^{1, p}(\Omega)\right)^{\prime} \text { for all } p>q .
$$

Proof. See Berger [3, Theorem 5.1].

We remark that the first version of this theorem was proved in [15] for the case $q=2, L=0$, as an extension of Murat's result [30]. Theorem 3.2.1 represents a generalization of [27, Lemma 3.1].

Now we prove the first convergence result:

3.2.5. Theorem. Let $\left\{u_{h}\right\}, h \in\left(0, h_{0}\right)$, be a family of physically admissible approximate transonic flow solutions. This means that for each $h \in\left(0, h_{0}\right)$, the approximation $u_{h}$ satisfies $(2.2 .16, \mathrm{a}-\mathrm{c})$ and $u_{h} \in \varphi_{s_{1}, K, h}^{a d}$. Then there exists a subsequence $\left\{u_{h_{n}}\right\}$ such that

$$
u_{h_{n}}^{\prime} \rightarrow u \text { strongly in } W^{1, p}(\Omega) \quad \forall p \in[1, \infty)
$$

(where $u_{h}^{\prime}$ is defined in (3.1.25)) and $u$ is a physically admissible solution of (1.2.14, a-c) satisfying $u \in \varphi_{s_{1}, K}^{a d}$. Moreover,

$$
\lim _{h_{n} \rightarrow 0}\left\|u_{c}-u_{h_{n}}\right\|_{W^{1, p}\left(\Omega_{h_{n}}\right)}=0, \quad p \in[1, \infty) .
$$

Proof. (I) Let $q \in(2, \infty)$ be arbitrary and fixed. Let us consider the subsequence $\left\{u_{h_{n}}\right\}$ and the function $u$ satisfying (3.1.33). For simplicity we shall write $h:=h_{n}$ in the sequel.

We define the linear functions $G, G_{h}: W^{1, q}(\Omega) \rightarrow \mathbb{R}^{1}$ :

$$
\begin{aligned}
& \text { (a) } G_{h}(v)=-\int_{\Omega_{h}} \nabla u_{h} \cdot \nabla r_{h} v d x-K \int_{\Omega_{h}} r_{h} v d x, \\
& v \in W^{1, q}(\Omega), h \in\left(0, h_{0}\right), \\
& \text { (b) } G(v)=-\int_{\Omega} \nabla u \cdot \nabla v d x-K \int_{\Omega} v d x, \quad v \in W^{1, q}(\Omega) .
\end{aligned}
$$

By the Hölder inequality, (3.1.27), where we set $s_{2}:=s_{1}$, and $(3.1 .20$, c), we 
get

$$
|G(v)|,\left|G_{h}(v)\right| \leq c\|v\|_{W^{1, q}(\Omega)}, \quad v \in W^{1, q}(\Omega), h \in\left(0, h_{0}\right)
$$

which means that $G, G_{h} \in\left(W^{1, q}(\Omega)\right)^{\prime}$.

(II) Now we show that

$$
G_{h} \rightarrow G \text { weakly in }\left(W^{1, q}(\Omega)\right)^{\prime}, \quad \text { if } h \rightarrow 0 .
$$

For arbitrary $v \in W^{1, q}(\Omega)$ we have

$$
\left|G_{h}(v)-G(v)\right| \leq I_{1}+I_{2}+I_{3},
$$

where

$$
\begin{aligned}
& I_{1}=\left|\int_{\Omega_{h}} \nabla u_{h} \cdot \nabla\left(r_{h} v-v_{c}\right) d x\right|+K\left|\int_{\Omega_{h}}\left(r_{h} v-v_{c}\right) d x\right|, \\
& I_{2}=\left|\int_{\Omega_{h}} \nabla u_{h} \cdot \nabla v_{c} d x-\int_{\Omega} \nabla u_{h}^{\prime} \cdot \nabla v d x\right|+K\left|\int_{\Omega_{h}} v_{c} d x-\int_{\Omega} v d x\right|, \\
& I_{3}=\left|\int_{\Omega} \nabla\left(u_{h}^{\prime}-u\right) \cdot \nabla v d x\right| .
\end{aligned}
$$

By (3.1.20, a), (3.1.27), and the Hölder inequality, we have $I_{1} \rightarrow 0$ for $h \rightarrow 0$. By (3.1.33) also $I_{3} \rightarrow 0$. Further,

$$
\begin{aligned}
I_{2} \leq & \left|\int_{\Omega_{h}} \nabla u_{h} \cdot \nabla v_{c} d x-\int_{\Omega} \nabla \bar{u}_{h} \cdot \nabla v d x\right| \\
& +K\left|\int_{\Omega_{h}} v_{c} d x-\int_{\Omega} v d x\right|+\left|\int_{\Omega} \nabla\left(u_{h}^{\prime}-\bar{u}_{h}\right) \cdot \nabla v d x\right|
\end{aligned}
$$

If we use (3.1.2), the Hölder inequality, (3.1.29), (3.1.30), and the absolute continuity of the Lebesgue integral, we find that

$$
I_{2} \leq \int_{\omega_{h} \cup \tau_{h}}\left|\nabla \bar{u}_{h} \cdot \nabla v_{c}\right| d x+K \int_{\omega_{h} \cup \tau_{h}}\left|v_{c}\right| d x+c h\|v\|_{W^{1, q}(\Omega)} \rightarrow 0 .
$$

Hence, (3.2.10) is valid.

Since the functions $u_{h}$ satisfy the discrete entropy condition, (2.2.20) and (2.2.21) hold. There exist constants $L \geq 0$ and $h_{1} \in\left(0, h_{0}\right)$ such that $\gamma(h) \leq L$ for all $h \in\left(0, h_{1}\right)$. For arbitrary $v \in C_{0}^{\infty}(\Omega)^{+}$we have $r_{h} v \in E_{h}^{+}$and thus, by $(3.1 .20, d)$ and $(3.2 .8, a)$, we get

$$
G_{h}(v) \leq L\|v\|_{L^{\infty}(\Omega)}, \quad v \in C_{0}^{\infty}(\Omega)^{+}, h \in\left(0, h_{1}\right)
$$

We see that the assumptions of Theorem 3.2.1 are satisfied, and since $q>2$ was arbitrary, it follows that

$$
G_{h} \rightarrow G \text { strongly in }\left(W^{1, p}(\Omega)\right)^{\prime} \text { for all } p>2 .
$$


(III) We use the relations obtained by a recurrent process:

(a) $\left|u-u_{h}^{\prime}\right|_{W^{1,2}(\Omega)}^{2}=\int_{\Omega} \nabla\left(u-u_{h}^{\prime}\right) \cdot \nabla\left(u-u_{h}^{\prime}\right) d x$

$$
=\overbrace{\int_{\Omega} \nabla u \cdot \nabla\left(u-u_{h}^{\prime}\right) d x}^{1}-\int_{\Omega} \nabla u_{h}^{\prime} \cdot \nabla\left(u-u_{h}^{\prime}\right) d x,
$$

(b) $\int_{\Omega} \nabla u_{h}^{\prime} \cdot \nabla\left(u-u_{h}^{\prime}\right) d x$

$$
\begin{aligned}
= & \overbrace{\int_{\Omega} \nabla u_{h}^{\prime} \cdot \nabla\left(u-\overline{r_{h} u}\right) d x}^{2}+\int_{\Omega} \nabla u_{h}^{\prime} \cdot \nabla\left(\overline{r_{h} u}-\bar{u}_{h}\right) d x \\
& +\overbrace{\int_{\Omega} \nabla u_{h}^{\prime} \cdot \nabla\left(\bar{u}_{h}-u_{h}^{\prime}\right) d x}^{3},
\end{aligned}
$$

(c) $\int_{\Omega} \nabla u_{h}^{\prime} \cdot \nabla\left(\overline{r_{h} u}-\bar{u}_{h}\right) d x$

$$
=\overbrace{\int_{\Omega} \nabla\left(u_{h}^{\prime}-\bar{u}_{h}\right) \cdot \nabla\left(\overline{r_{h} u}-\bar{u}_{h}\right) d x}^{4}+\int_{\Omega} \nabla \bar{u}_{h} \cdot \nabla\left(\overline{r_{h} u}-\bar{u}_{h}\right) d x,
$$

(d) $\int_{\Omega} \nabla \bar{u}_{h} \cdot \nabla\left(\overline{r_{h} u}-\bar{u}_{h}\right) d x$

$$
=\int_{\Omega_{h}} \nabla u_{h} \cdot \nabla\left(r_{h} u-u_{h}\right) d x
$$

$$
+\overbrace{\int_{\omega_{h}} \nabla \bar{u}_{h} \cdot \nabla\left(\overline{r_{h} u}-\bar{u}_{h}\right) d x-\int_{\tau_{h}} \nabla u_{h} \cdot \nabla\left(r_{h} u-u_{h}\right) d x}^{5},
$$

(e) $-\int_{\Omega_{h}} \nabla u_{h} \cdot \nabla\left(r_{h} u-u_{h}\right) d x$

$$
\begin{aligned}
= & G_{h}\left(u-\bar{u}_{h}\right)+K \int_{\Omega_{h}}\left(r_{h} u-u_{h}\right) d x \\
= & \overbrace{G\left(u-u_{h}^{\prime}\right)}^{6}+\overbrace{G\left(u_{h}^{\prime}-\bar{u}_{h}\right)}^{7}+\overbrace{\left(G_{h}-G\right)\left(u-\bar{u}_{h}\right)}^{8} \\
& +K \int_{\Omega}\left(\bar{r}_{h} u-\bar{u}_{h}\right) d x
\end{aligned}
$$

$$
+\overbrace{K \int_{\tau_{h}}\left(r_{h} u-u_{h}\right) d x-K \int_{\omega_{h}}\left(\overline{r_{h} u}-\bar{u}_{h}\right) d x}^{9},
$$

(f) $\int_{\Omega}\left(\overline{r_{h} u}-\bar{u}_{h}\right) d x=\overbrace{\int_{\Omega}\left(\overline{r_{h} u}-u\right) d x}^{10}+\overbrace{\int_{\Omega}\left(u-u_{h}^{\prime}\right) d x}^{11}$

$$
+\overbrace{\int_{\Omega}\left(u_{h}^{\prime}-\bar{u}_{h}\right) d x}^{12}
$$


All the terms in (3.2.12) denoted 1-12 tend to zero, if $h \rightarrow 0$. This is a consequence of the following table, where we introduce the formulas and estimates which imply the convergence of the particular terms 1-12:

1. (3.1.33)

2. $(3.1 .31),(3.1 .20, \mathrm{~b})$

3. $(3.1 .31),(3.1 .30)$

4. $(3.1 .20, \mathrm{e}),(3.1 .29),(3.1 .30)$

5. (3.1.20, c and e), (3.1.27), (3.1.28), (3.1.2)

6. (3.1.33)

7. (3.2.9), (3.1.27), (3.1.30)

8. (3.1.28), (3.2.11)

9. (3.1.20, c and e), (3.1.29), (3.1.2)

10. $(3.1 .20, \mathrm{~b})$

11. $(3.1 .33)$

12. $(3.1 .30)$.

Hence, $\left|u-u_{h}^{\prime}\right|_{W^{1,2}(\Omega)} \rightarrow 0$ if $h \rightarrow 0$. From (3.1.33) and the compact imbedding $W^{1,2}(\Omega) \hookrightarrow \hookrightarrow L^{2}(\Omega)$ it follows that $\left\|u-u_{h}^{\prime}\right\|_{L^{2}(\Omega)} \rightarrow 0$ and thus,

$$
\lim _{h \rightarrow 0}\left\|u-u_{h}^{\prime}\right\|_{W^{1,2}(\Omega)}=0 .
$$

The sequence $u_{h}^{\prime}$ is bounded in $W^{1, \infty}(\Omega)$. By the Lebesgue theorem, (3.2.13) implies

$$
\lim _{h \rightarrow 0}\left\|u-u_{h}^{\prime}\right\|_{W^{1, p}(\Omega)}=0, \quad p \in[1, \infty) .
$$

Further, from the relations

$$
\begin{aligned}
\left\|u_{h}-u_{c}\right\|_{W^{1, p}\left(\Omega_{h}\right)}^{p} & =\left\|u_{h}-u\right\|_{W^{1, p}\left(\Omega-\omega_{h}\right)}^{p}+\left\|u_{h}-u_{c}\right\|_{W^{1, p}\left(\tau_{h}\right)}^{p}, \\
\left\|u_{h}-u_{c}\right\|_{W^{1, p}\left(\tau_{h}\right)} & \leq\left\|u_{h}\right\|_{W^{1, p}\left(\tau_{h}\right)}+\left\|u_{c}\right\|_{W^{1, p}\left(\tau_{h}\right)}, \\
\left\|u_{h}-u_{c}\right\|_{W^{1, p}\left(\Omega-\omega_{h}\right)} & \leq\left\|\bar{u}_{h}-u\right\|_{W^{1, p}(\Omega)} \\
& \leq\left\|\bar{u}_{h}-u_{h}^{\prime}\right\|_{W^{1, p}(\Omega)}+\left\|u_{h}^{\prime}-u\right\|_{W^{1, p}(\Omega)},
\end{aligned}
$$

(3.1.4), (3.1.5), (3.2.14), (3.1.29), (3.1.30), and (3.1.2) we obtain (3.2.7).

(IV) Now we show that $u$ is a physical solution of problem $(2.2 .16$, a-c).

(a) Let $\varepsilon>0$ be an arbitrary number. Then by (3.1.31) (where $s_{2}:=s_{1}$ ), there exists $h_{\ell} \in\left(0, h_{0}\right)$ such that

$$
\left\|\nabla u_{h}^{\prime}\right\|_{L^{\infty}(\Omega)} \leq \sqrt{s_{1}}+\varepsilon \quad \forall h \in\left(0, h_{\varepsilon}\right) .
$$

By virtue of (3.2.6) we can extract a subsequence from $\left\{\nabla u_{h}^{\prime}\right\}$ which converges to $\nabla u$ a.e. in $\Omega$. Hence, we have $\|\nabla u\|_{L^{\infty}(\Omega)} \leq \sqrt{s_{1}}+\varepsilon$ for each $\varepsilon>0$ and thus,

$$
\|\nabla u\|_{L^{\infty}(\Omega)}^{2} \leq s_{1} .
$$

(b) Let $v \in \mathscr{E}^{+}$(see (1.2.19)). Then $r_{h} v \in E_{h}^{+}$, and by (2.2.20), (3.1.20, d), and $(3.2 .8, a)$,

$$
G_{h}(v) \leq \gamma(h)\|v\|_{L^{\infty}(\Omega)} .
$$

Using the strong convergence $G_{h} \rightarrow G$ in $\left(W^{1, p}(\Omega)\right)^{\prime} \quad(p \in(2, \infty))$ and (2.2.21), we immediately get from $(3.2 .17)$ the relation

$$
G(v)=-\int_{\Omega} \nabla u \cdot \nabla v d x-K \int_{\Omega} v d x \leq 0,
$$

which is (1.2.22). Thus, $u \in \varphi_{s_{1}}^{a d}$. 
(c) In order to prove that $u$ satisfies $\left(1.2 .14\right.$, a-c), we observe that $u_{h}^{\prime}-\bar{u}_{h}^{*}=$ $\hat{z}_{h} \in V, u_{h}^{\prime} \rightarrow u, \bar{u}_{h}^{*} \rightarrow u^{*}$ and thus, $\hat{z}_{h} \rightarrow z:=u-u^{*}$ in $W^{1,2}(\Omega)$. Since $V$ is a closed subspace of $W^{1,2}(\Omega)$, we have $u-u^{*} \in V$, which is $(1.2 .14, \mathrm{~b})$.

(d) For the verification of $(1.2 .14, a, c)$ we shall consider an arbitrary $v \in \mathscr{V}$ and denote $v_{h}=\Pi_{h} v \in V_{h}$. Using (2.2.16, c), (1.2.11), (2.2.1), and (3.1.15, $a-b)$, we can write

$$
\begin{aligned}
a\left(u_{h}^{\prime}, \hat{v}_{h}\right) & +\left[a\left(u_{h}^{\prime}, \bar{v}_{h}\right)-a\left(u_{h}^{\prime}, \hat{v}_{h}\right)\right]+\left[a\left(\bar{u}_{h}, \bar{v}_{h}\right)-a\left(u_{h}^{\prime}, \bar{v}_{h}\right)\right] \\
& +\left[\int_{\tau_{h}} b \rho\left(\left|\nabla u_{h}\right|^{2}\right) \nabla u_{h} \cdot \nabla v_{h} d x-\int_{\omega_{h}} b \rho\left(\left|\nabla \bar{u}_{h}\right|^{2}\right) \nabla \bar{u}_{h} \cdot \nabla \bar{v}_{h} d x\right] \\
& +\left[a_{h}\left(u_{h}, v_{h}\right)-\tilde{a}_{h}\left(u_{h}, v_{h}\right)\right] \\
= & L\left(\bar{v}_{h}\right)+\left[\widetilde{L}_{h}\left(v_{h}\right)-L\left(\bar{v}_{h}\right)\right]+\left[L_{h}\left(v_{h}\right)-\tilde{L}_{h}\left(v_{h}\right)\right]+\mathscr{H}_{h}\left(v_{h}\right) .
\end{aligned}
$$

In view of Lemma 3.1.22, we have $\bar{v}_{h}, \hat{v}_{h} \rightarrow v$ in $W^{1, p}(\Omega)$ for $p>2$, and hence also in $W^{1,2}(\Omega)$. From this, Lemma 1.2.13, (2.2.14), and the fact that $u_{h}^{\prime} \rightarrow u$, it follows that

$$
\begin{gathered}
\lim _{h \rightarrow 0} a\left(u_{h}^{\prime}, \hat{v}_{h}\right)=a(u, v), \\
\lim _{h \rightarrow 0} L\left(\bar{v}_{h}\right)=L(v), \quad \lim _{h \rightarrow 0} \mathscr{H}_{h}\left(v_{h}\right)=0 .
\end{gathered}
$$

Using Lemma 1.2.13, (3.1.17), (3.1.30), (3.1.4), the boundedness of $\bar{u}_{h}, \bar{v}_{h}$ in $W^{1,2}\left(\Omega \cup \Omega_{h}\right),(2.2 .12),(2.2 .13)$, and (3.1.3), we find that all terms in the square brackets in (3.2.18) tend to zero if $h \rightarrow 0$. This, and (3.2.19), immediately imply that the relation $a(u, v)=L(v)$ is satisfied for all $v \in \mathscr{V}$. Now from the density of $\mathscr{V}$ in $V$ and the properties of $a(u, \cdot)$ and $L$ we see that $(1.2 .14, \mathrm{a}, \mathrm{c})$ holds.

3.2.20. Corollary. If there exists only one physically admissible solution $u \in$ $\varphi_{s_{1}, K}^{a d}$ of problem $(1.2 .14, \mathrm{a}-\mathrm{c})$, then we do not need to confine ourselves to subsequences of the family $\left\{u_{h}\right\}_{h \in\left(0, h_{0}\right)}$, and we have

$$
\lim _{h \rightarrow 0}\left\|u_{h}^{\prime}-u\right\|_{W^{1, p}(\Omega)}=\lim _{h \rightarrow 0}\left\|u_{h}-u_{c}\right\|_{W^{1, p}\left(\Omega_{h}\right)}=0
$$

for $p \in[1, \infty)$.

3.2.21. Remark. The assumptions on approximate solutions may seem rather strong, but they are necessary to guarantee their physical admissibility. In the following, Theorem 3.2.5 will be used as a tool for proving the convergence of solutions of the minimization problems.

3.3. Convergence of solutions of the minimization problems. In this subsection we shall deal with the convergence of the solutions of the minimization problems (2.3.5). For this purpose we must introduce the Ritz projection $P_{h}: W^{1,2}(\Omega) \rightarrow$ $u_{h}^{*}+V_{h}$ defined by

(a) $P_{h} u \in X_{h}$,

(b) $P_{h} u-u_{h}^{*} \in V_{h}$,

(c) $\int_{\Omega_{h}} \nabla P_{h} u \cdot \nabla v_{h} d x=\int_{\Omega_{h}} \nabla u_{c} \cdot \nabla v_{h} d x \quad \forall v_{h} \in V_{h}$. 
3.3.2. Lemma. Provided $u \in u^{*}+V, u^{*} \in W^{1, \infty}(\Omega)$, and $u_{h}^{*}=r_{h} u^{*}$, we have

(a) $\lim _{h \rightarrow 0}\left|P_{h} u-u_{c}\right|_{W^{1,2}\left(\Omega_{h}\right)}=0$,

(b) $\lim _{h \rightarrow 0}\left|\overline{P_{h} u}-u\right|_{W^{1,2}(\Omega)}=0$,

(c) $\left\|P_{h} u\right\|_{W^{1,2}\left(\Omega_{h}\right)},\left\|\overline{P_{h} u}\right\|_{W^{1,2}(\Omega)} \leq c \quad \forall h \in\left(0, h_{0}\right)$.

Proof. Let $u \in u^{*}+V$. Then we have $u=u^{*}+z$ with $z \in V, P_{h} u=u_{h}^{*}+z_{h}$ with $z_{h} \in V_{h}$, and for any $\chi_{h} \in V_{h}$ we obtain

$$
\begin{aligned}
\left|P_{h} u-u_{c}\right|_{W^{1,2}\left(\Omega_{h}\right)}^{2}= & \int_{\Omega_{h}} \nabla\left(P_{h} u-u_{c}\right) \cdot \nabla\left(u_{h}^{*}-u_{c}^{*}\right) d x \\
& +\int_{\Omega_{h}} \nabla\left(P_{h} u-u_{c}\right) \cdot \nabla\left(z_{h}-\chi_{h}\right) d x \\
& +\int_{\Omega_{h}} \nabla\left(P_{h} u-u_{c}\right) \cdot \nabla\left(\chi_{h}-z_{c}\right) d x .
\end{aligned}
$$

This, the Cauchy inequality, the fact that $z_{h}-\chi_{h} \in V_{h}$, and $(3.3 .1, \mathrm{c})$ yield the relation

$$
\left|P_{h} u-u_{c}\right|_{W^{1,2}\left(\Omega_{h}\right)} \leq\left|u_{h}^{*}-u^{*}\right|_{W^{1,2}\left(\Omega_{h}\right)}+\inf _{\chi_{h} \in V_{h}}\left|\chi_{h}-z_{c}\right|_{W^{1,2}\left(\Omega_{h}\right)} .
$$

By $(3.1 .20, a)$, the density of $\mathscr{V}$ in the space $V$, and the property $(3.1 .23, a)$ we get the assertion $(3.3 .3, a)$. The result $(3.3 .3, b)$ is obtained on the basis of (3.1.2), (3.1.4), and the absolute continuity of the Lebesgue integral (cf. (3.2.15)).

Further, using the relation $P_{h} u=u_{h}^{*}+z_{h}, z_{h} \in V_{h},(3.3 .1, \mathrm{c})$ with $v_{h}:=$ $z_{h},(3.1 .8)$, the boundedness of the family $\left\{u_{h}^{*}\right\}$, and (3.1.4), (3.1.5), we get $(3.3 .3, \mathrm{c})$.

We now formulate our second fundamental convergence result.

3.3.4. Theorem. Let the following assumptions be satisfied:

(a) Problem (1.2.14, a-c) has exactly one solution $u \in \varphi_{s_{1}, K}^{\text {ad }}$ for some $K \geq 0$. No other solution of $(1.2 .14$, a-c) satisfying the entropy condition (1.2.22) exists.

(b) There exists a constant $s_{2} \geq s_{1}$ such that

$$
\left\|\nabla P_{h} u\right\|_{L^{\infty}\left(\Omega_{h}\right)}^{2} \leq s_{2}, \quad h \in\left(0, h_{0}\right) .
$$

(c) We choose $\varepsilon \in\left[1, \frac{3}{2}\right)$.

(d) The family $\left\{\mathscr{T}_{h}\right\}_{h \in\left(0, h_{0}\right)}$ is quasiuniform.

Then the solutions $\left\{u_{h}\right\}$ of the minimization problems (2.3.5) converge to $u$ in the following sense:

$$
\begin{gathered}
\lim _{h \rightarrow 0}\left\|u_{h}^{\prime}-u\right\|_{W^{1, p}(\Omega)}=0, \\
\lim _{h \rightarrow 0}\left\|u_{h}-u_{c}\right\|_{W^{1, p}\left(\Omega_{h}\right)}=0,
\end{gathered}
$$

where $u_{h}^{\prime}$ was defined in (3.1.25) and $p \in[1, \infty)$.

Proof. (I) We shall show that all $u_{h}, h \in\left(0, h_{0}\right)$, satisfy $(2.2 .16)$ with an appropriate choice of the functional $\mathscr{X}_{h}$ with property $(2.2 .14)$. Let us put

$$
\mathscr{X}_{h}\left(v_{h}\right)=a_{h}\left(u_{h}, v_{h}\right)-L_{h}\left(v_{h}\right), \quad v_{h} \in V_{h} .
$$


By (2.3.3) we have

$$
\mathscr{X}_{h}\left(v_{h}\right)=\int_{\Omega_{h}} \nabla \xi_{h}\left(u_{h}\right) \cdot \nabla v_{h} d x, \quad v_{h} \in V_{h},
$$

and in order to prove (2.2.14), it suffices to show that

$$
\left|\xi_{h}\left(u_{h}\right)\right|_{W^{1,2}\left(\Omega_{h}\right)} \rightarrow 0 \text { if } h \rightarrow 0 .
$$

From the definition of the minimization problem (2.3.5) and assumption (3.3.5) we obtain the following inequality:

$$
\begin{aligned}
0 & \leq \mathscr{I}_{h}\left(u_{h}\right)=\frac{1}{2}\left|\xi_{h}\left(u_{h}\right)\right|_{W^{1,2}\left(\Omega_{h}\right)}^{2}+\mathscr{P}_{h}\left(u_{h}\right)=\min _{\substack{\varphi_{h} \in u_{h}^{*}+V_{h} \\
\left\|\nabla \varphi_{h}\right\|_{L^{\infty}\left(\Omega_{h}\right)}^{2} \leq s_{2}}} \mathscr{I}_{h}\left(\varphi_{h}\right) \\
& \leq \mathscr{I}_{h}\left(P_{h} u\right)=\frac{1}{2}\left|\xi_{h}\left(P_{h} u\right)\right|_{W^{1,2}\left(\Omega_{h}\right)}^{2}+\mathscr{P}_{h}\left(P_{h} u\right) .
\end{aligned}
$$

(1) First, we shall deal with the term $\mathscr{P}_{h}\left(P_{h} u\right)$. By (2.3.4) we have

$$
\mathscr{P}_{h}\left(P_{h} u\right)=\frac{\mu}{2} \sum_{P_{i} \in \hat{\sigma}_{h}} \frac{1}{A_{i}^{\varepsilon}}\left[\left(-\int_{\Omega_{h}} \nabla P_{h} u \cdot \nabla w_{h_{i}} d x-K \int_{\Omega_{h}} w_{h_{i}} d x\right)^{+}\right]^{2}
$$

For $P_{i} \in \hat{\sigma}_{h}$ let us consider the expression

$$
I_{i}:=-\int_{\Omega_{h}} \nabla P_{h} u \cdot \nabla w_{h_{i}} d x-K \int_{\Omega_{h}} w_{h_{i}} d x .
$$

If $\Gamma_{D} \neq \varnothing$, then $w_{h_{i}} \in V_{h}$, and by the definition of $P_{h}$ we have

$$
I_{i}=-\int_{\Omega_{h}} \nabla u_{c} \cdot \nabla w_{h_{i}} d x-K \int_{\Omega_{h}} w_{h_{i}} d x .
$$

However, the same is valid if $\Gamma_{D}=\varnothing$, because

$$
\tilde{w}_{h_{i}}:=w_{h_{i}}-\frac{1}{\operatorname{meas}\left(\Omega_{h}\right)} \int_{\Omega_{h}} w_{h_{i}} d x \in V_{h}
$$

and $\nabla \tilde{w}_{h_{i}}=\nabla w_{h_{i}}$.

Now, in view of (3.3.14), we can write

$$
I_{i}=I_{i}^{1}+I_{i}^{2}+I_{i}^{3}
$$

where

$$
\begin{aligned}
I_{i}^{1}= & -\int_{\Omega} \nabla u \cdot \nabla I_{h}^{\mathrm{id}} \bar{w}_{h_{i}} d x-K \int_{\Omega} I_{h}^{\mathrm{id}} \bar{w}_{h_{i}} d x \\
I_{i}^{2}= & \int_{\Omega} \nabla u \cdot \nabla\left(I_{h}^{\mathrm{id}} \bar{w}_{h_{i}}-\bar{w}_{h_{i}}\right) d x+K \int_{\Omega}\left(I_{h}^{\mathrm{id}} \bar{w}_{h_{i}}-\bar{w}_{h_{i}}\right) d x \\
I_{i}^{3}= & \int_{\omega_{h}} \nabla u \cdot \nabla \bar{w}_{h_{i}} d x+K \int_{\omega_{h}} \bar{w}_{h_{i}} d x \\
& -\int_{\tau_{h}} \nabla u_{c} \cdot \nabla w_{h_{i}} d x-K \int_{\tau_{h}} w_{h_{i}} d x .
\end{aligned}
$$

It is evident that $w_{h_{i}} \in E_{h}^{+}$for $P_{i} \in \hat{\sigma}_{h}$ and, by (3.1.14), $I_{h}^{\mathrm{id}} w_{h_{i}} \in E^{+}$. Hence, since $u \in \varphi_{s_{1}, K}^{a d}$, we have

$$
I_{i}^{1} \leq 0
$$


Further, from the quasiuniformity of the family $\left\{\mathscr{T}_{h}\right\}_{h \in\left(0, h_{0}\right)},(2.3 .7),(2.3 .8)$, (3.1.13), and the assumption that $\|\nabla u\|_{L^{\infty}(\Omega)}^{2} \leq s_{1}$, it follows that

$$
\begin{aligned}
\left|I_{i}^{2}\right| & \leq \sqrt{s_{1}} \int_{\Omega}\left|\nabla\left(I_{h}^{\mathrm{id}} \bar{w}_{h_{i}}-\bar{w}_{h_{i}}\right)\right| d x+K \int_{\Omega}\left|I_{h}^{\mathrm{id}} \bar{w}_{h_{i}}-\bar{w}_{h_{i}}\right| d x \\
& \leq \operatorname{ch}\left\|w_{h_{i}}\right\|_{W^{1,1}\left(\Omega_{h}\right)} \leq \operatorname{ch} \frac{A_{i}}{h} \leq c h^{2}, \quad h \in\left(0, h_{0}\right) .
\end{aligned}
$$

Using (2.2.4), we deduce that

$$
\operatorname{meas}\left[\left(\omega_{h} \cup \tau_{h}\right) \cap \operatorname{supp} \bar{w}_{h_{i}}\right] \leq c h^{3},
$$

which together with (2.3.7) yields the estimate

$$
\text { (3.3.20) }\left|I_{i}^{3}\right| \leq\left(\frac{c}{h}+c\right) \operatorname{meas}\left[\left(\omega_{h} \cup \tau_{h}\right) \cap \operatorname{supp} \bar{w}_{h_{i}}\right] \leq c h^{2}, \quad h \in\left(0, h_{0}\right) .
$$

Of course, if $P_{i}$ is not a vertex of a triangle $T \in \mathscr{T}_{h}$ adjacent to $\partial \Omega_{h}$, then

$$
I_{i}^{2}=I_{i}^{3}=0
$$

By (3.3.15)-(3.3.21) we have

$$
\begin{aligned}
I_{i}^{+} & \leq\left(I_{i}^{1}\right)^{+}+\left(I_{i}^{2}\right)^{+}+\left(I_{i}^{3}\right)^{+} \\
& \leq \begin{cases}c h^{2} & \text { if } P_{i} \in \hat{\sigma}_{h} \cap T \text { and } T \in \mathscr{T}_{h} \text { is a triangle adjacent to } \partial \Omega_{h}, \\
0 & \text { otherwise. }\end{cases}
\end{aligned}
$$

Set $N_{h}^{*}=\operatorname{card}\left\{P_{i} \in \hat{\sigma}_{h} ; P_{i} \in T\right.$, where $T \in \mathscr{T}_{h}$ is a triangle adjacent to $\left.\partial \Omega_{h}\right\}$. As $\left\{\mathscr{T}_{h \in\left(0, h_{0}\right)}\right.$ is quasiuniform, there holds

$$
N_{h}^{*} \leq \frac{c}{h}
$$

Now, from (2.3.8), (3.3.12), (3.3.13), (3.3.14), (3.3.22), (3.3.23), and assumption $(c)$, we obtain the estimate

$$
\mathscr{P}_{h}\left(P_{h} u\right) \leq c \frac{\mu}{2} N_{h}^{*} h^{-2 \varepsilon} h^{4} \leq c h^{3-2 \varepsilon} \rightarrow 0 \quad \text { if } h \rightarrow 0 .
$$

(2) In the following we shall consider the expression

$$
\left|\xi_{h}\left(P_{h} u\right)\right|_{W^{1,2}\left(\Omega_{h}\right)} \text { as } h \rightarrow 0 .
$$

Using Lemma 3.1.7, (2.3.3, a-b), (1.2.14, a-c), (1.2.11), and (2.2.1), we get for 
$\xi_{h}:=\xi_{h}\left(P_{h} u\right)$ the estimate

$$
\begin{aligned}
& \left\|\xi_{h}\right\|_{W^{1,2}\left(\Omega_{h}\right)}^{2} \leq c\left|\xi_{h}\right|_{W^{1,2}(\Omega)}^{2}=\int_{\Omega_{h}} \nabla \xi_{h} \cdot \nabla \xi_{h} d x \\
& \quad=a_{h}\left(P_{h} u, \xi_{h}\right)-L_{h}\left(\xi_{h}\right)-a\left(u, \hat{\xi}_{h}\right)+L\left(\hat{\xi}_{h}\right) \\
& \quad \leq \overbrace{\left|a_{h}\left(P_{h} u, \xi_{h}\right)-\tilde{a}_{h}\left(P_{h} u, \xi_{h}\right)\right|}^{1}+\overbrace{\left|\int_{\tau_{h}} b \rho\left(\left|\nabla P_{h} u\right|^{2}\right) \nabla P_{h} u \cdot \nabla \xi_{h} d x\right|}^{2}
\end{aligned}
$$

$$
\begin{aligned}
& +\overbrace{\left|\int_{\omega_{h}} b \rho\left(\left|\nabla \overline{P_{h} u}\right|^{2}\right) \nabla \overline{P_{h} u} \cdot \nabla \bar{\xi}_{h} d x\right|}^{3}+\overbrace{\left|a\left(\overline{P_{h} u}, \bar{\xi}_{h}\right)-a\left(\overline{P_{h} u}, \hat{\xi}_{h}\right)\right|}^{4} \\
& +\overbrace{\left|a\left(\overline{P_{h} u}, \hat{\xi}_{h}\right)-a\left(u, \hat{\xi}_{h}\right)\right|}^{5}+\overbrace{\left|L_{h}\left(\xi_{h}\right)-\tilde{L}_{h}\left(\xi_{h}\right)\right|}^{6} \\
& +\overbrace{\left|\widetilde{L}_{h}\left(\xi_{h}\right)-L\left(\bar{\xi}_{h}\right)\right|}^{7}+\overbrace{\left|L\left(\bar{\xi}_{h}\right)-L\left(\hat{\xi}_{h}\right)\right|}^{8} .
\end{aligned}
$$

The terms in (3.3.25) denoted by $1-4$ and 6-8 are bounded by $c h\left\|\xi_{h}\right\|_{W^{1,2}\left(\Omega_{h}\right)}$, as we can prove on the basis of the following table with the relations which yield the particular estimates:

1. $(2.2 .12),(3.3 .3, \mathrm{c})$

2.,3. (1.1.8, c), 1.2.3(A), Cauchy inequality, (3.1.4), (3.3.3, c)

4. 1.2.13 a, (3.1.17), (3.3.3, c)

6. $(2.2 .13)$

7. $(3.1 .3)$

8. 1.2.13 d, (3.1.17).

We next estimate the fifth term. In view of $1.2 .13 \mathrm{c}$, this term is bounded by

$$
c\left|\overline{P_{h} u}-u\right|_{W^{1,2}(\Omega)}|\hat{\xi}|_{W^{1,2}(\Omega)} \text {. }
$$

Further,

$$
\begin{aligned}
& \left|\hat{\xi}_{h}\right|_{W^{1,2}(\Omega)} \leq\left|\hat{\xi}_{h}-\bar{\xi}_{h}\right|_{W^{1,2}(\Omega)}+\left|\bar{\xi}_{h}\right|_{W^{1,2}(\Omega)}, \\
& \left|\bar{\xi}_{h}\right|_{W^{1,2}(\Omega)}^{2} \leq\left|\xi_{h}\right|_{W^{1,2}\left(\Omega_{h}\right)}^{2}+\left|\bar{\xi}_{h}\right|_{W^{1,2}\left(\omega_{h}\right)}^{2} .
\end{aligned}
$$

From this, (3.1.17) and (3.1.4) we get a bound of term 5 in the form

$$
c\left|\overline{P_{h} u}-u\right|_{W^{1,2}(\Omega)}\left\|\xi_{h}\right\|_{W^{1,2}\left(\Omega_{h}\right)} .
$$

Summarizing the above results, we have

$$
\left\|\xi_{h}\right\|_{W^{1,2}\left(\Omega_{h}\right)}^{2} \leq c\left(h+\left|\overline{P_{h} u}-u\right|_{W^{1,2}(\Omega)}\right)\left\|\xi_{h}\right\|_{W^{1,2}\left(\Omega_{h}\right)}
$$

and hence, by virtue of $(3.3 .3, b)$,

$$
\left\|\xi_{h}\left(P_{h} u\right)\right\|_{W^{1,2}\left(\Omega_{h}\right)} \rightarrow 0 \quad \text { if } h \rightarrow 0 \text {. }
$$

(3) The relations in (3.3.11), (3.3.24), and (3.3.26) imply that

$$
\frac{1}{2}\left|\xi_{h}\left(u_{h}\right)\right|_{W^{1,2}\left(\Omega_{h}\right)}^{2}+\mathscr{P}_{h}\left(u_{h}\right) \rightarrow 0 \text { if } h \rightarrow 0 .
$$

This means that (3.3.10) is valid and thus, $\left\{u_{h}\right\}_{h \in\left(0, h_{0}\right)}$ is a family of solutions of $(2.2 .16, \mathrm{a}-\mathrm{c})$ with $\mathscr{H}_{h}$ satisfying $(2.2 .14)$. 
(II) Now we come to the entropy condition. From (3.3.27), assumption (c), and Theorem 2.3.10 it follows that the functions $u_{h}$ satisfy the entropy inequality $(2.2 .20)$ with the function

$$
\gamma(h):=\sqrt{\mathscr{P}_{h}\left(u_{h}\right)} h^{\varepsilon-1},
$$

which has property (2.2.21). Finally, we apply Theorem 3.2.5 (where we substitute $s_{1}:=s_{2}$ ) and Corollary 3.2.20, and we immediately get (3.3.6), (3.3.7).

3.3.28. Remark. By a more involved technique it is possible to extend Theorem 3.3.4 to the case of a regular family of triangulations, provided it satisfies the conditions $N_{h} \approx 1 / h^{2}$ and $N_{h}^{*} \approx 1 / h$.

In the case $\Gamma_{D}=\varnothing$ the main results remain valid if instead of the spaces $\mathscr{V}$ and $V_{h}$ defined in $(1.2 .7, \mathrm{~b})$ and $(2.1 .6, \mathrm{~b})$ we use

$$
\mathscr{V}=\left\{v \in C^{\infty}(\bar{\Omega}) ; \int_{\partial \Omega} v d S=0\right\}
$$

and

$$
V_{h}=\left\{v \in X_{h} ; \int_{\partial \Omega_{h}} v_{h} d S=0\right\}
$$

respectively.

3.3.29. Remark. Concerning assumption (3.3.5), we refer to the result of Rannacher and Scott [33], where they prove the stability of the Ritz projection in $W^{1, p}(\Omega)$ for all $p \in[2, \infty]$. According to the authors of [33], it is possible to extend this stability result to more general boundary conditions and to the case of a piecewise smooth boundary, provided the corner angles do not exceed some critical values.

3.3.30. Remark. In the proof of Theorem 3.3.4 it has been shown that the limit case $\varepsilon=1$ is still allowed. This is an improvement of the results of Berger [3] achieved by our refined analysis. Nevertheless, since the function $\gamma(h)=\sqrt{\mathscr{P}_{h}\left(u_{h}\right)} h^{\varepsilon-1}$ determines how well $u_{h}$ satisfies the entropy condition, the choice $\varepsilon>1$ seems to be more suitable. For $\varepsilon>1$ the convergence $\gamma(h) \rightarrow 0$ is faster than for $\varepsilon=1$, when $\gamma(h)$ can tend to zero very slowly. On the other hand, owing to the variational crimes, it is not possible to choose $\varepsilon \geq 1.5$ in domains with curved boundaries.

The numerical experiments in calculations of transonic flow past NACA 0012 profile are in agreement with our theoretical results. They show that the choice $\varepsilon=1$ gives a too flat velocity distribution along the profile. If we take $\varepsilon>1$, the results are better and closer to the results obtained by finite difference methods (cf., e.g., [3]). However, the choice $\varepsilon \geq 1.5$ leads in some cases to instabilities and slow convergence of the iterative process for obtaining an approximate solution. If we choose $\varepsilon \in(1,1.5)$, these problems disappeared.

\section{BIBLIOGRAPHY}

1. B. Arlinger, Axisymmetric transonic flow computations using a multigrid method, Lecture Notes in Phys., vol. 141, Springer-Verlag, Berlin and New York, 1981, pp. 55-60.

2. H. Berger, Finite-Element-Approximationen für transonische Strömungen, Dr. rer. nat. Dissertation, Universität Stuttgart, 1989. 
3. _ A convergent finite element formulation for transonic flow, Numer. Math. 56 (1989), 425-447.

4. H. Berger, G. Warnecke, and W. Wendland, Finite elements for transonic flows, Numer. Methods Partial Differential Equations 6 (1990), 17-42.

5. M. O. Bristeau, R. Glowinski, J. Periaux, P. Perrier, O. Pironneau, and G. Poirier, Application of optimal control and finite element methods to the calculation of transonic flows and incompressible viscous flows, Numerical Methods in Applied Fluid Dynamics (B. Hunt, ed.), Academic Press, New York, 1980, pp. 203-312.

6. P. G. Ciarlet, The finite element method for elliptic problems, North-Holland, Amsterdam, 1979.

7. P. G. Ciarlet and P. A. Raviart, General Lagrange and Hermite interpolation in $\mathbb{R}^{n}$ with applications to finite element methods, Arch. Rational Mech. Anal. 46 (1972), 177-199.

8. J. D. Cole and E. M. Murman, Calculation of plane steady transonic flows, AIAA J. 9 (1971), 199-206.

9. H. Deconinck, The numerical computation of transonic potential flows, Ph.D. thesis, Vriji Universiteit, Brussel, 1983.

10. H. Deconinck and C. Hirsch, Transonic flow calculations with higher finite elements, Lecture Notes in Phys., vol. 141, Springer-Verlag, Berlin and New York, 1981, pp. 138-143.

11. R. DiPerna, Convergence of the viscosity method for isentropic gas dynamics, Comm. Math. Phys. 91 (1983), 1-30.

12. P. Doktor, On the density of smooth functions in certain subspaces of Sobolev space, Comment. Math. Univ. Carolinae 14 (1973), 609-622.

13. M. Feistauer, On irrotational flows through cascades of profiles in a layer of variable thickness, Apl. Mat. 29 (1984), 423-458.

14. _ On the finite element approximation of a cascade flow problem, Numer. Math. 50 (1987), 655-684.

15. M. Feistauer, J. Mandel, and J. Nečas, Entropy regularization of the transonic potential flow problems, Comment. Math. Univ. Carolinae 25 (1984), 431-443.

16. M. Feistauer and J. Nečas, On the solvability of transonic potential flow problems, Z. Anal. Anwendungen 4 (1985), 305-329.

17. _ Viscosity method in a transonic flow, Comm. Partial Differential Equations 13 (1988), 775-812.

18. _ Remarks on the solvability of transonic flow problems, Manuscripta Math. 61 (1988), 417-428.

19. M. Feistauer and V. Sobotíková, Finite element approximation of nonlinear elliptic problems with discontinuous coefficients, $\mathrm{M}^{2} \mathrm{AN} 24$ (1990), 457-500.

20. M. Feistauer and A. Ženišsek, Finite element solution of nonlinear elliptic problems, Numer. Math. 50 (1987), 665-684.

21. Compactness method in the finite element theory of nonlinear elliptic problems, Numer. Math. 52 (1988), 147-163.

22. R. Glowinski, Lectures on numerical methods for nonlinear variational problems, SpringerVerlag, Berlin and New York, 1981.

23. __ Numerical methods for nonlinear variational problems, Springer-Verlag, Berlin and New York, 1984.

24. R. Glowinski and O. Pironneau, On the computation of transonic flows, Functional Analysis and Numerical Analysis (H. Fujita, ed.), Japan Soc. for the Promotion of Science, 1978, pp. 143-173.

25. A. Jameson, Acceleration of transonic potential flow calculations on arbitrary meshes by the multiple grid method, AIAA Paper 79-1458 (1979).

26. A. Kufner, O. John, and S. Fučik, Function spaces, Academia, Praha, 1977.

27. J. Mandel and J. Nečas, Convergence of finite elements for transonic potential flows, SIAM J. Numer. Anal. 24 (1987), 985-996. 
28. R. Mani and A. J. Acosta, Quasi two-dimensional flows through a cascade, Trans. ASME Ser. A, J. Engrg. for Power 90 (1968), No. 2.

29. C. Morawetz, On a weak solution for a transonic flow problem, Comm. Pure Appl. Math. 38 (1985), 797-818.

30. F. Murat, L'injection du cône positif de $H^{-1}$ dans $W^{-1, q}$ est compacte pour tout $q<2$, J. Math. Pures Appl. (9) 60 (1981), 309-322.

31. J. Nečas, Les méthodes directes en théorie des équations Elliptiques, Masson, Paris, 1967.

32. __ Ecoulements de fluide: compacité par entropie, Masson, Paris, 1988.

33. R. Rannacher and R. Scott, Some optimal error estimates for piecewise linear finite element approximations, Math. Comp. 38 (1982), 437-445.

34. G. Strang, Variational crimes in the finite element method, The Mathematical Foundations of the Finite Element Method with Applications to Partial Differential Equations (A. K. Aziz, ed.), Academic Press, New York, 1972, pp. 689-710.

35. A. Ženíšek, Discrete forms of Friedrichs' inequalities in the finite element method, RAIRO Numer. Anal. 15 (1981), 265-286.

36. M. Zlámal, Curved elements in the finite element method. I, SIAM J. Numer. Anal. 10 (1973), 229-240.

University of Stuttgart, Mathematical Institute A, Pfaffenwaldring 57, D-7000 StutTGaRT 80, GermanY

Charles University Prague, Mathematical Institute, Sokolovska' 83, 18600 Praha 8, Czech Republic

E-mail address: feist@cspguk11.bitnet 